Pacific Journal of Mathematics

ON THE EIGENVALUES OF REPRESENTATIONS OF
REFLECTION GROUPS AND WREATH PRODUCTS 


\title{
ON THE EIGENVALUES OF REPRESENTATIONS OF REFLECTION GROUPS AND WREATH PRODUCTS
}

\author{
JOHN R. STEMbRIDGE
}

\begin{abstract}
Let $G$ be a finite group. The eigenvalues of any $g \in G$ of order $m$ in a (complex) representation $\rho$ may be expressed in the form $\omega^{e_{1}}, \omega^{e_{2}}, \ldots$, with $\omega=e^{2 \pi i / m}$. We call the integers $e_{j}(\bmod m)$ the cyclic exponents of $g$ with respect to $\rho$. We give explicit combinatorial descriptions of the cyclic exponents of the (irreducible) representations of the symmetric groups, the classical Weyl groups, and certain finite unitary reflection groups. We also show that for any finite group $G$, the cyclic exponents of the wreath product $G$ ? $S_{n}$ can be described in terms of the cyclic exponents of $G$. For each of the infinite families of finite unitary reflection groups $W$, we also provide explicit, combinatorial descriptions of the generalized exponents of $W$. These parameters arise in the symmetric algebra of the associated reflection representation, and by a theorem of Springer, are closely related to the cyclic exponents of $W$.
\end{abstract}

0. Introduction. Let $G$ be a finite group acting on a complex vector space $V$. If $g \in G$ is an element of order $m$, then the eigenvalues of $g$ on $V$ are $m$ th roots of unity, and may therefore be expressed in the form $\omega^{e_{1}}, \omega^{e_{2}}, \ldots$, with $\omega=e^{2 \pi i / m}$. We call the integers $e_{j}(\bmod$ $m$ ) the cyclic exponents of $g$ with respect to $V$. This terminology is partly inspired by the case in which $G$ is a Weyl group and $V$ carries the reflection representation of $G$. If $g \in G$ is a Coxeter element, then the corresponding cyclic exponents $e_{j}$, reduced $\bmod m$ to the form $0 \leq e_{j}<m$, are the classical exponents of $G[\mathrm{~B}]$.

The central objective of this paper is to provide explicit descriptions of cyclic exponents for groups $G$ whose irreducible representations have intrinsic combinatorial structure. In the typical situation, we have a group $G$ acting (irreducibly) on some space $V$ whose dimension is in one-to-one correspondence with a set $C$ of combinatorial objects. (The prototype we have in mind is the case in which $G=S_{n}$ and $C$ is a set of standard Young tableaux.) The problem to be solved is to find, for each $g \in G$, a "natural" rule for attaching an exponent $e=e_{g}(c)$ to each of the combinatorial objects $c \in C$, so that $\left\{\omega^{e_{g}(c)}: c \in C\right\}$ is the multiset of eigenvalues of $g$ on $V$. 
In addition to the purely combinatorial interest in cyclic exponents, there are a number of situations in which specific exponents for specific groups arise naturally. In the context of real or unitary reflection groups $G$, there is an especially elegant example of this phenomenon due to Springer [Sp]. For each irreducible character $\chi$ of $G$, there exist $n=\operatorname{deg} \chi$ integer parameters $e_{1}, \ldots, e_{n}$ called generalized exponents that describe certain structural properties of the symmetric algebra of the reflection representation of $G$. (See $\S 2$ for definitions). According to Springer's work, if $g \in G$ is $\omega$-regular of order $m\left(\omega=e^{2 \pi i / m}\right)$, then the cyclic exponents of $g$ corresponding to $\chi$ are the generalized exponents $\bmod m$.

In this paper we restrict our attention to two types of groups: (1) The various classical series (i.e., infinite families) of real or unitary reflection groups, and (2) wreath products of the form $G \backslash S_{n}$, where $G$ is an arbitrary finite group. The irreducible representations of these groups do have intrinsic combinatorial structure, and so it is possible to formulate the description of their cyclic exponents as a combinatorial problem. Of course, a treatment of (1) independent of the classification of reflection groups would be desirable, but the irreducible representations of the exceptional groups do not seem to be amenable to combinatorial analysis.

A secondary objective of this paper will be to provide explicit descriptions of generalized exponents for each of the classical series of reflection groups. We will also give explicit formulas for the associated generating functions. In the Weyl group case, these generating functions are the "fake degrees" of the corresponding finite Chevalley groups $[\mathbf{C}, \mathbf{L}]$, for which there are known formulas due to Steinberg [S1] (type $A$ ), and Lusztig [L] (types $B$ and $D$ ). Also, we note that the generalized exponents of type $A$ are known and were originally described by Stanley [St1, 4.11].

The remainder of this paper is organized as follows. In $\S 1$ we give a few general facts about eigenvalues and cyclic exponents in finite groups. In $\S 2$ we outline the connections between cyclic and generalized exponents in reflection groups. Since the ideas behind Springer's theorem will be useful later, we have included a sketch of the proof. In $\S 3$ we construct the cyclic and generalized exponents of the symmetric groups. Included is a proof of Stanley's description of the generalized exponents since it plays a key role in the techniques that follow. In $\S 4$ we consider the wreath products $G ? S_{n}$; the main results of this section are Theorems 4.6 and 4.7 which provide explicit descriptions 
of the cyclic exponents of $G \nmid S_{n}$ in terms of those of $G$. In $\S 5$, we consider the reflection groups $\left.C_{m}\right\} S_{n}$, where $C_{m}$ denotes the cyclic group of order $m$. Of course, the cyclic exponents in this case may be obtained as a corollary of the results of $\S 4$, but a further problem to be addressed is the description of the generalized exponents and the associated generating functions. This will include the fake degrees of type $B$ mentioned above. In $\S 6$, we consider the remaining infinite family of irreducible, unitary reflection groups; these are indexed by three parameters $d, m, n$ with $d \mid m$ and may be realized as subgroups of index $m / d$ in $C_{m}>S_{n}$. (See $\S 6$ for definitions). We again give descriptions and generating functions for the generalized exponents. Included as a special case $(m=2, d=1)$ are the fake degrees of type $D$. We do not attempt a complete description of the cyclic exponents of these groups, since the techniques we will develop would only produce an indirect description in these cases. However, for the special case corresponding to the Weyl groups $D_{n}$, we have included a complete description of the cyclic exponents since they have a relatively simple form.

In the appendix (§7), we have included two theorems which we have been unable to find in the literature: a recurrence for the evaluation of irreducible characters of $G\left\{S_{n}\right.$ analogous to the Murnaghan-Nakayama rule, and formulas that relate the so-called "difference characters" of $D_{n}$ to the character table of the symmetric group on $n / 2$ letters. Although the construction of the character table of $D_{n}$ is attributed to Young [Y], one finds that his description of the difference characters is inaccurate, or at best, vague and misleading. (See especially p. 288 of QSA V).

Conventions. All vector spaces and algebras to be considered will use the complex field. All groups to be considered will be finite and all representations finite-dimensional (or at least graded). We use the notation $I_{G}$ for the set of irreducible characters of the group $G$ and $C_{G}$ for the set of conjugacy classes. If the vector space $V$ carries a linear representation of $G$, we write char $V$ for the character of $G$ on $V$. The notation $\langle\cdot, \cdot\rangle_{G}$ is reserved for the usual Hermitian inner product on the space of $G$-class functions; namely,

$$
\langle f, g\rangle_{G}=\frac{1}{|G|} \sum_{x \in G} f(x) \bar{g}(x) .
$$

The subscript $G$ may be dropped if it is clear from the context. 
The notation $\mathscr{P}$ is used for the set of partitions; i.e., integer sequences of the form $\lambda=\left(\lambda_{1} \geq \lambda_{2} \geq \cdots \geq \lambda_{l}>0\right)$ for some $l \geq 0$ called the length of $\lambda$. We write $\ell(\lambda)=l,|\lambda|=\sum \lambda_{l}$, and if $|\lambda|=n$, then $\lambda$ is said to be a partition of $n$.

Acknowledgments. I would like to thank Professors R. Steinberg and R. Stanley for valuable discussions. I would also like to credit the manuscript of Kraśkiewicz and Weyman [KW] for providing part of the motivation for the questions addressed in this paper.

1. Preliminaries. Before beginning the combinatorial analysis of the cyclic exponents of specific groups, we first collect a few general observations valid for arbitrary finite groups. Continuing the setting of the introduction, let $g \in G$ be of order $m$, and let the eigenvalues of the action of $g$ on $V$ be $\omega^{e_{1}}, \omega^{e_{2}}, \ldots$, where $\omega=e^{2 \pi i / m}$. Form the generating function

$$
P_{V, g}(q)=q^{e_{1}}+q^{e_{2}}+\cdots
$$

for the cyclic exponents, where $q$ is an indeterminate. Note that $P_{V, g}$ is well-defined $\bmod 1-q^{m}$. Since $P_{V, g}$ depends only on the character $\chi$ of $V$ (and the conjugacy class of $g$ ), we will usually write $P_{\chi, g}$ in place of $P_{V, g}$.

Note that $P_{\chi, g}(\omega)=\chi(g)$. More generally, the eigenvalues of $g^{r}$ on $V$ are $\omega^{r e_{1}}, \omega^{r e_{2}}, \ldots$, so we also have $P_{\chi, g}\left(\omega^{r}\right)=\chi\left(g^{r}\right)$. By reduction of $P_{\chi, g}(q) \bmod 1-q^{m}$, we may assume $\operatorname{deg} P_{\chi, g}<m$, and therefore, the values of $P_{\chi, g}(q)$ at $q=\omega^{r}(0 \leq r<m)$ uniquely determine $P_{\chi, g}$. That is, we may regard the calculation of cyclic exponents as an interpolation problem. In summary, we have

Proposition 1.1. If $P(q) \in \mathbf{C}[q]$ satisfies $P\left(\omega^{r}\right)=\chi\left(g^{r}\right)$ for $0 \leq r<$ $m$, then $P=P_{\chi, g} \bmod \left(1-q^{m}\right)$.

Another interpretation of the cyclic exponents of $g$ can be obtained via representations of $G$ induced from the cyclic subgroup $\langle g\rangle$. Let $\varphi^{r}:\langle g\rangle \rightarrow \mathbf{C}$ denote the irreducible $\langle g\rangle$-character defined by $\varphi^{r}(g)=$ $\omega^{r}$, and let $\varphi^{r} \uparrow G$ denote the resulting induced $G$-character.

PROPOSITION 1.2.

$$
\sum_{r=0}^{m-1} q^{r}\left(\varphi^{r} \uparrow G\right)=\sum_{\chi \in I_{G}} P_{\chi, g}(q) \chi \quad\left(\bmod 1-q^{m}\right) .
$$


Proof. We may assume $\operatorname{deg} P_{\chi, g}<m$ without loss of generality. In that case, the coefficient of $q^{r}$ in $P_{\chi, g}$ is the dimension of the eigenspace corresponding to $\omega^{r}$ in the action of $g$ on a $G$-module of character $\chi$. However, by Frobenius reciprocity, the coefficient of $\chi$ in $\varphi^{r} \uparrow G$ is also the coefficient of $\varphi^{r}$ in the restriction $\chi \downarrow\langle g\rangle$. Since $\langle g\rangle$-modules of character $\varphi^{r}$ may be identified with $\omega^{r}$-eigenvectors of $g$, the claim follows.

This result shows that the multiplicity of $\chi$ in $\varphi^{r} \uparrow G$ is the number of times that $r(\bmod m)$ occurs as a cyclic exponent of $g$ (with respect to $\chi$ ).

It is interesting to note that in the case $G=S_{n}, g=n$-cycle, there have been natural occurrences of $S_{n}$-modules isomorphic to $\varphi \uparrow S_{n}$ in the recent literature. For example, Stanley [St2, §7] has shown that such a structure occurs (modulo the sign character) in the homology of the partition lattice $\Pi_{n}$, and Joyal $[\mathbf{J}]$ and Garsia [G] have found such a structure in the free Lie algebra on $n$ generators.

2. Exponents in reflection groups. A reflection (or pseudo-reflection) is defined to be a linear transformation of finite order whose fixed point set is a hyperplane; a reflection group is a (finite) subgroup of a general linear group $\mathrm{GL}(V)$ generated by reflections. In this section we consider the case in which $G$ acts as a reflection group on $V=\mathbf{C}^{n}$.

Let $S=\bigoplus_{k \geq 0} S^{k}(V)$ denote the symmetric algebra of $V$, and let $S^{G}$ denote the graded subalgebra of $G$-invariants. By a theorem of Chevalley [Ch] (proved originally only for real reflection groups), it is known that $S^{G}$ is a polynomial ring generated by $n$ algebraically independent homogeneous elements (see also [ST] and [St1]). We refer to the degrees $d_{1}, \ldots, d_{n}$ of these generators as the homogeneous degrees of the reflection group $G$. Among the many properties of these degrees, we mention in particular the fact that

$$
|G|=d_{1} d_{2} \cdots d_{n}
$$

Among the many sources for proofs, we cite [B, Ch, St1, ST].

The homogeneous degrees are also closely related to certain cyclic exponents of $G$. Consider first the case of Coxeter elements in Weyl groups. We recall that a Coxeter element $g$ in a Weyl group $G$ may be defined as the product of the simple reflections, taken in any order; all such products are conjugate in $G$ (see $[\mathbf{B}, \mathbf{S 2}])$. As we remarked in the introduction, the classical exponents $e_{1}, \ldots, e_{n}$ of $G$ are, in our terminology, the cyclic exponents of such a $g$ on $V$, reduced $\bmod m$ to 
the form $0 \leq e_{j}<m$. On a case-by-case basis, Coxeter [Co] observed that $e_{j}=d_{j}-1$. A uniform proof was later given by Coleman [Col].

Springer [Sp] found a generalization of this observation valid for regular elements in arbitrary reflection groups. A vector $v \in V$ is said to be regular if it is not contained in any of the hyperplanes defined by the reflections in $G$, and $g \in G$ is said to be regular if it has a regular eigenvector. If $g$ has a regular eigenvector with eigenvalue $\omega \in \mathbf{C}$, then $g$ is said to be $\omega$-regular.

THEOREM 2.1 (Springer [Sp, (4.2v)]). If $g \in G$ is $\omega$-regular, then the eigenvalues of $g$ on $V$ are $\omega^{-\left(d_{1}-1\right)}, \ldots, \omega^{-\left(d_{n}-1\right)}$.

By a theorem of Coxeter [S2], one knows that a Coxeter element has a regular eigenvector with eigenvalue $e^{-2 \pi i / m}$, so this result does generalize the fact that $e_{j}=d_{j}-1$ in Weyl groups.

Springer has also shown that there is a more general relationship between the $G$-module structure of the symmetric algebra $S=S(V)$ and the cyclic exponents of regular $g$. To explain this relationship, let $J=\bigoplus_{k \geq 0} J^{k}$ denote the graded, $G$-stable ideal of $S$ generated by the invariants in $S^{G}$ of positive degree, and for each character $\chi \in I_{G}$, let $G_{\chi}(q)$ denote the Poincare series of the $\chi$ th isotypic component of $S / J=\bigoplus_{k \geq 0} S^{k} / J^{k}$. One may obtain the generating functions $G_{\chi}(q)$ via the expansion

$$
\sum_{k \geq 0} q^{k} \operatorname{char}\left(S^{k} / J^{k}\right)=\sum_{\chi \in I_{G}} G_{\chi}(q) \chi
$$

i.e., the coefficient of $q^{k}$ in $G_{\chi}(q)$ is the multiplicity of $\chi$ in $S^{k} / J^{k}$. In case $G$ is a Weyl group, the series $G_{\chi}(q)$ are the fake degrees of the corresponding Chevalley groups; tables of these polynomials can be found in [C, 13.8].

Define the generalized $\chi$-exponents of $G$ to be the integers $e_{1}(\chi)$, $e_{2}(\chi), \ldots$ that appear in the expansion

$$
G_{\chi}(q)=q^{e_{1}(\chi)}+q^{e_{2}(\chi)}+\cdots .
$$

The number of such exponents is $\operatorname{deg} \chi$, since the $G$-module structure of $S / J$ is isomorphic to the regular representation of $G$ [Ch] (see also [St1, 4.10]).

We are now ready to state Springer's result.

THEOREM 2.2 (Springer [Sp, (4.5)]). Let $g \in G$ be $\omega^{s}$-regular of order $m$, where $\omega=e^{2 \pi i / m}$. We have

$$
G_{\chi}\left(q^{s}\right)=P_{\chi, g}(q)\left(\bmod 1-q^{m}\right) .
$$


In particular, if $g$ is $\omega$-regular, then the generalized $\chi$-exponents of $G$ $(\bmod m)$ are the cyclic exponents of $g$ with respect to $\chi$.

REMARKS. (1) This version is dual to the formulation in [Sp], since we have chosen to work with the symmetric algebra of $V$ rather than the dual $V^{*}$.

(2) The eigenvalues corresponding to the regular eigenvectors of any $g \in G$ must have the same order as $g$ [Sp, (4.2i)]. Consequently, if $g \in G$ is $\omega^{s}$-regular, then some power $g^{r}$ is $\omega$-regular.

Sketch of Proof. The previous remark allows us to restrict our attention to the case $s=1$; i.e., assume $g$ is $\omega$-regular and $\omega=e^{2 \pi i / m}$. In view of (2.2) and Proposition 1.2, it therefore suffices to show

$$
\sum_{k \geq 0} q^{k} \operatorname{char}\left(S^{k} / J^{k}\right)=\sum_{r=0}^{m-1} q^{r}\left(\varphi^{r} \uparrow G\right) \quad\left(\bmod 1-q^{m}\right) .
$$

To evaluate the (graded) character of $S / J$ at $h \in G$, observe that if the eigenvalues of $h$ on $V$ are $\alpha_{i}=\alpha_{i}(h)(1 \leq i \leq n)$, then the eigenvalues of $h$ on $S^{k}$ are the monomials of degree $k$ in $\alpha_{1}, \ldots, \alpha_{n}$. Therefore,

$$
\sum_{k \geq 0} q^{k} \operatorname{char}\left(S^{k}\right)(h)=\prod_{i=1}^{n} \frac{1}{1-\alpha_{i} q}=\frac{1}{\operatorname{det}(1-q h)},
$$

where $\operatorname{det}(1-q h)$ is interpreted in $\operatorname{End}_{\mathbf{C}[q]}(V)$. The various isotypic components of $S$ are known to be free as $S^{G}$-modules (e.g., [St1, 3.10]), so we have

$$
S \cong S^{G} \otimes S / J
$$

as graded $G$-modules. Since the Poincaré series of $S^{G}$ is $\Pi\left(1-q^{d_{l}}\right)^{-1}$, it follows that

$$
\sum_{k \geq 0} q^{k} \operatorname{char}\left(S^{k} / J^{k}\right)(h)=\prod_{i=1}^{n} \frac{1-q^{d_{i}}}{1-\alpha_{i}(h) q},
$$

or equivalently via (2.2),

$$
\sum_{\chi \in I_{G}} G_{\chi}(q) \chi(h)=\operatorname{det}(1-q h)^{-1} \prod_{i=1}^{n}\left(1-q^{d_{i}}\right) .
$$

Now consider the induced character $\sum q^{r}\left(\varphi^{r} \uparrow G\right)$. Evaluated at $q=\omega^{j}$, the orthogonality relations for cyclic group characters imply

$$
\sum_{r=0}^{m-1} q^{r} \varphi^{r}\left(g^{i}\right)= \begin{cases}m & \text { if }-j=i \bmod m \\ 0 & \text { otherwise }\end{cases}
$$


Using standard properties of induced characters (e.g., [CR, (10.9)]), it follows that

$$
\sum_{r=0}^{m-1} q^{r}\left(\varphi^{r} \uparrow G\right)(h)= \begin{cases}z_{h} & \text { if } h \sim g^{-j} \\ 0 & \text { otherwise }\end{cases}
$$

where $q=\omega^{j}, \sim$ denotes conjugacy in $G$, and $z_{h}$ denotes the size of the $G$-centralizer of $h$. Thus, to establish (2.3), it suffices to prove

$$
\prod_{i=1}^{n} \frac{1-q^{d_{i}}}{1-\alpha_{i}(h) q}= \begin{cases}z_{h} & \text { if } h \sim g^{-j} \\ 0 & \text { otherwise }\end{cases}
$$

for all $h \in G$ and all choices $q=\omega^{j}(0 \leq j<m)$.

For simplicity, we consider only the case $q=\omega$.

The numerator of (2.4) has a zero of order $\left|\left\{i: m \mid d_{i}\right\}\right|$ at $q=\omega$, whereas the denominator has a zero of order $\operatorname{dim} V\left(h, \omega^{-1}\right)$, where $V(h, \alpha)$ denotes the eigenspace of $h$ on $V$ corresponding to the eigenvalue $\alpha$. Since (2.4) is a polynomial in $q$ (recall that $S / J$ is isomorphic to the regular representation of $G$ ), it follows that

$$
\operatorname{dim} V\left(h, \omega^{-1}\right) \leq\left|\left\{i: m \mid d_{i}\right\}\right| .
$$

This observation is strengthened in [Sp, (4.2ii,iv)], where it is proved that equality occurs iff $h \sim g^{-1}$. This proves $(2.6 \mathrm{~b})$.

Finally, note that the eigenvalues of $g$ acting on $V$ are $\omega^{-\left(d_{1}-1\right)}, \ldots$, $\omega^{-\left(d_{n}-1\right)}$, by Theorem 2.1. Therefore,

$$
\lim _{q \rightarrow \omega} \prod_{i=1}^{n} \frac{1-q^{d_{i}}}{1-\alpha_{i}\left(g^{-1}\right) q}=\lim _{q \rightarrow \omega} \prod_{i=1}^{n} \frac{1-q^{d_{i}}}{1-\omega^{d_{i}-1} q}=\prod_{m \mid d_{i}} d_{i} .
$$

However, the $G$-centralizer $C_{G}(g)$ is itself a reflection group, acting on $V(g, \omega)$, with homogeneous degrees $\left\{d_{i}: m \mid d_{i}\right\}$ [Sp, (4.2iii)]. Thus, (2.6a) now follows from the application of $(2.1)$ to the reflection group $C_{G}(g)$.

\section{The symmetric groups.}

A. Background. The irreducible representations of $S_{n}$ are indexed by partitions of $n$ [JK]. Associated with a partition $\lambda$ is the diagram

$$
D_{\lambda}=\left\{(i, j) \in \mathbf{Z}^{2}: 1 \leq i \leq \ell(\lambda), 1 \leq j \leq \lambda_{i}\right\},
$$

which we regard as an array of cells in the plane with matrix-style coordinates. 


$$
\begin{array}{lllll}
1 & 1 & 2 & 4 & 8 \\
2 & 4 & 5 & 5 & \\
8 & 9 & & &
\end{array}
$$

\section{FIGURE 1}

A tableau of shape $\lambda$ is an assignment $T: D_{\lambda} \rightarrow \mathbf{P}$ of positive integers to the cells of $D_{\lambda}$ so that

$$
\begin{aligned}
& \text { (1) } T(i, j) \leq T(i, j+1) \quad \text { (nondecreasing rows), } \\
& \text { (2) } T(i, j)<T(i+1, j) \quad \text { (increasing columns). }
\end{aligned}
$$

A reverse tableau is an assignment $T: D_{\lambda} \rightarrow \mathbf{P}$ with the inequalities reversed; i.e., nonincreasing rows and decreasing columns. A tableau of shape $(5,4,2)$ appears in Figure 1 ; a reverse tableau appears in Figure 2(a). The content of a tableau $T$ is the sequence $\gamma(T)=\left(\gamma_{1}, \gamma_{2}, \ldots\right)$, where

$$
\gamma_{k}=\left|\left\{(i, j) \in D_{\lambda}: T(i, j)=k\right\}\right| \text {. }
$$

Recall that the Schur functions $s_{\lambda}$ may be described as generating functions for tableaux. Specifically, we have

$$
s_{\lambda}\left(x_{1}, x_{2}, \ldots\right)=\sum_{T: D_{\lambda} \rightarrow \mathbf{P}} x^{\gamma(T)},
$$

summed over tableaux $T$, where $x^{\gamma}=x_{1}^{\gamma_{1}} x_{2}^{\gamma_{2}} \cdots$ [M1]. We remark that since the Schur functions are symmetric with respect to permutations of the variables $x_{i}$ [M1], we may alternatively regard $s_{\lambda}$ as the analogous generating function for reverse tableaux.

In our description of the cyclic exponents of $S_{n}$, it will be convenient to exploit properties of the $S_{n}$-characters and tableaux indexed by skew partitions. A general skew partition (of size $n$ ) is indicated by the notation $\lambda / \mu$, where $\lambda$ and $\mu$ are any pair of partitions with $D_{\lambda} \supseteq D_{\mu}$ (and $\left.|\lambda|-|\mu|=n\right)$. The diagram associated with $\lambda / \mu$ is the array $D_{\lambda / \mu}:=D_{\lambda}-D_{\mu}$, and a tableau of shape $\lambda / \mu$ is an assignment $T: D_{\lambda / \mu} \rightarrow \mathbf{P}$ satisfying the usual rules in (3.1). The corresponding generating function for tableaux of shape $\lambda / \mu$, namely,

$$
s_{\lambda / \mu}\left(x_{1}, x_{2}, \ldots\right)=\sum_{T: D_{\lambda / \mu} \rightarrow \mathbf{P}} x^{\gamma(T)}
$$

is said to be a skew Schur function.

A tableau of content $(1,1, \ldots, 1)$ is said to be standard; we use the notation $\mathscr{F}^{\lambda}$ (resp., $\mathscr{F}^{\lambda / \mu}$ ) for the set of standard tableaux of shape $\lambda$ (resp., $\lambda / \mu$ ). If $k+1$ appears in a row strictly below $k$ in a standard tableau $T$, then $k$ is said to be a descent of $T$, and we write $D(T)$ for 


\begin{tabular}{|c|c|c|c|c|c|}
\hline 109 & 2 & 2 & 1 & 2 & 4 \\
\hline 7 & 6 & & 3 & 6 & 7 \\
\hline 5 & 0 & & 5 & 8 & 13 \\
\hline 1 & & & 9 & 12 & \\
\hline
\end{tabular}

(a) reverse

FIGURE 2

(b) standard

the set of descents in $T$. The index of $T$ is defined to be the sum of the descents; i.e.,

$$
\text { ind } T=\sum_{k \in D(T)} k
$$

In the example in Figure 2(b), the descent set is $\{2,4,7,8,11\}$ and the index is 32 .

Let $I_{\lambda / \mu}(q)$ denote the generating function for the indices of the standard tableaux of shape $\lambda / \mu$ (possibly $\mu=\varnothing$ ); i.e.,

$$
I_{\lambda / \mu}(q)=\sum_{T \in \mathscr{F} \lambda / \mu} q^{\text {ind } T}
$$

The following result connects the indices of standard tableaux of shape $\lambda / \mu$ to the principal specialization of Schur functions. Although it is a straightforward application of Stanley's theory of $P$-partitions (and a proof may be found in [M1, pp.49-50]), we have included another proof which will serve as a model for similar arguments we will use in analyzing the generalized exponents of the reflection groups in $\S 6$.

LEMMA 3.1. Let $\lambda / \mu$ be a skew partition of $n$. We have

$$
s_{\lambda / \mu}\left(1, q, q^{2}, \ldots\right)=\frac{1}{(q)_{n}} I_{\lambda / \mu}(q),
$$

where $(q)_{n}=(1-q)\left(1-q^{2}\right) \cdots\left(1-q^{n}\right)$.

Proof. Interpret $s_{\lambda / \mu}\left(1, q, q^{2}, \ldots\right)$ as the generating function for reverse tableaux of shape $\lambda / \mu$, weighted according to the sum of the entries, with 0 as an allowable entry. Given such a tableau $T$, linearly order the cells of $D_{\lambda / \mu}$ as follows:

$$
(i, j)<\left(i^{\prime}, j^{\prime}\right) \text { iff }\left\{\begin{array}{l}
T(i, j)>T\left(i^{\prime}, j^{\prime}\right) \text {, or } \\
T(i, j)=T\left(i^{\prime}, j^{\prime}\right) \text { and } j<j^{\prime} .
\end{array}\right.
$$

By numbering the cells of $D_{\lambda / \mu}$ from 1 to $n$ according to this order, we obtain a standard tableau $S$. For example, Figure $2($ b) depicts the standard tableau obtained via this process from the reverse tableau in Figure 2(a). 
Let $\alpha_{i}$ denote the entry of $T$ in the cell numbered $i$ by $S$. Given $S$ and the sequence $\alpha$, it is easy to recover $T$, so the map $T \mapsto(S, \alpha)$ is injective. For fixed $S$, the possible sequences $\alpha$ which arise from this correspondence are characterized by the fact that $\alpha_{1} \geq \cdots \geq \alpha_{n} \geq 0$ and

$$
\alpha_{k}>\alpha_{k+1} \quad \text { if } \quad k \in D(S) \quad(1 \leq k<n) .
$$

Given such a sequence $\alpha$, subtract 1 from each of $\alpha_{1}, \ldots, \alpha_{k}$ for each $k \in D(S)$. The result is a partition $\alpha^{*}$ whose terms are characterized by the fact that $\alpha_{1}^{*} \geq \cdots \geq \alpha_{n}^{*} \geq 0$, and whose size satisfies $|\alpha|=$ $\left|\alpha^{*}\right|+$ ind $S$. Thus, $\alpha^{*}$ is an arbitrary partition of length at most $n$, and we may conclude that the generating function for the reverse tableaux $T$ corresponding to a fixed $S$ is of the form

$$
\sum_{\ell\left(\alpha^{*}\right) \leq n} q^{\left|\alpha^{*}\right|+\text { ind } S}=\frac{q^{\text {ind } S}}{(q)_{n}} .
$$

By adding the contributions corresponding to the various choices for $S$, we obtain the desired result.

B. Generalized exponents. Let $\chi^{\lambda}$ denote the irreducible $S_{n}$-character indexed by $\lambda$, and let $G_{\lambda}(q)$ denote the associated generating function for the generalized exponents corresponding to $\chi^{\lambda}$, as in $\S 2$. The following result, due independently to Lusztig and Stanley, describes the generalized exponents in combinatorial terms. We have included a proof since it is difficult to find in the literature (although a statement of the result can be found in [St1, 4.11]).

THEOREM 3.2. We have $G_{\lambda}(q)=I_{\lambda}(q)$; i.e., the indices of the standard tableaux of shape $\lambda$ are the generalized exponents of $S_{n}$ corresponding to $\chi^{\lambda}$.

Proof. Regard $S_{n}$ as a reflection group acting on $\mathbf{C}^{n}$ by permuting the standard coordinates. The homogeneous degrees with respect to this (reducible) representation of $S_{n}$ are $1,2, \ldots, n$, and so (2.5) may be rewritten as

$$
\frac{(q)_{n}}{\operatorname{det}(1-q w)}=\sum_{|\lambda|=n} \chi^{\lambda}(w) G_{\lambda}(q)
$$

for any $w \in S_{n}$.

Note that if $w$ is of cycle-type $\mu=\left(\mu_{1}, \mu_{2}, \ldots\right)$, then the characteristic polynomial of $w$ acting on $\mathbf{C}^{n}$ is of the form

$$
\operatorname{det}(1-q w)=\prod_{i}\left(1-q^{\mu_{i}}\right) .
$$


Using $p_{r}=p_{r}\left(x_{1}, x_{2}, \ldots\right)=x_{1}^{r}+x_{2}^{r}+\cdots$ to denote the $r$ th power sum, (3.2) implies

$$
(q)_{n} p_{\mu}\left(1, q, q^{2} \ldots\right)=\sum_{|\lambda|=n} \chi^{\lambda}(w) G_{\lambda}(q),
$$

where $p_{\mu}$ is an abbreviation for $p_{\mu_{1}} p_{\mu_{2}} \cdots$. One may deduce $G_{\lambda}(q)=$ $(q)_{n} s_{\lambda}\left(1, q, q^{2}, \ldots\right)$ by comparing this with the Frobenius expansion [M1, (7.8)]

$$
p_{\mu}\left(x_{1}, x_{2}, \ldots\right)=\sum_{|\lambda|=n} \chi^{\lambda}(w) s_{\lambda}\left(x_{1}, x_{2}, \ldots\right) .
$$

Apply Lemma 3.1 to complete the proof.

We remark that the series $s_{\lambda}\left(1, q, q^{2}, \ldots\right)$ has an explicit factorization of the form

$$
s_{\lambda}\left(1, q, q^{2}, \ldots\right)=\frac{q^{n(\lambda)}}{H_{\lambda}(q)}=q^{n(\lambda)} \prod_{(i, j) \in D_{\lambda}}\left(1-q^{h(i, j)}\right)^{-1},
$$

where $n(\lambda)=\sum_{i}(i-1) \lambda_{i}$ and $h(i, j)$ is the hooklength at $(i, j)$. (See [M1, p.28] for details). Consequently, we have the explicit formula

$$
G_{\lambda}(q)=I_{\lambda}(q)=q^{n(\lambda)} \frac{(q)_{n}}{H_{\lambda}(q)} .
$$

C. Cyclic exponents. Let $\mu$ be a partition of $n$, and let $m=$ $\left[\mu_{1}, \mu_{2}, \ldots\right]$ (least common multiple) denote the order of any $w \in S_{n}$ of cycle-type $\mu$. Partition the integers $1,2, \ldots, n$ into $\ell(\mu)$ consecutive blocks of sizes $\mu_{1}, \mu_{2}, \ldots$, and let $b_{\mu}=\left(b_{\mu}(1), \ldots, b_{\mu}(n)\right)$ denote the sequence defined as follows. If $j(1 \leq j \leq n)$ is the $i$ th smallest element assigned to the $k$ th block, we define $b_{\mu}(j)=i m / \mu_{k}$. Thus, we have

$$
b_{\mu}=\left(\frac{m}{\mu_{1}}, \frac{2 m}{\mu_{1}}, \ldots, m, \frac{m}{\mu_{2}}, \frac{2 m}{\mu_{2}}, \ldots, m, \ldots\right) .
$$

For example, $b_{(4,4,3,2)}=(3,6,9,12,3,6,9,12,4,8,12,6,12)$. For any standard tableau $T$, we define the $\mu$-index via

$$
\operatorname{ind}_{\mu}(T)=\sum_{k \in D(T)} b_{\mu}(k)(\bmod m) .
$$

The $(4,4,3,2)$-index of the tableau in Figure $2(\mathrm{~b})$ is $3 \bmod 12$.

Let $P_{\lambda, \mu}(q)$ denote the cyclic exponent generating function corresponding to any $w \in S_{n}$ of cycle-type $\mu$, acting on the irreducible $S_{n}$-module indexed by $\lambda$. The following result, conjectured by Stanley [St3], describes these exponents in combinatorial terms. 
THEOREM 3.3. We have

$$
P_{\lambda, \mu}(q)=\sum_{T \in \mathscr{F} \lambda} q^{\text {ind }_{\mu}(T)}\left(\bmod 1-q^{m}\right)
$$

i.e., the cyclic exponents of $w$ with respect to $\lambda$ are the $\mu$-indices of the standard tableaux of shape $\lambda$.

As a first step towards the proof of Theorem 3.3, let $\chi^{\lambda / \nu}$ denote the character corresponding to the skew-representation of $S_{n}$ indexed by $\lambda / \nu$. If $|\lambda|=n+k,|\nu|=k$, then $\chi^{\lambda / \nu}$ may be characterized indirectly via the branching rule

$$
\chi^{\lambda} \downarrow S_{k} \times S_{n}=\sum_{|\nu|=k} \chi^{\nu} \times \chi^{\lambda / \nu},
$$

where $\chi_{1} \times \chi_{2}$ denotes the outer tensor product of $S_{k}$-and $S_{n}$-characters. One may take (3.4) as the definition of the skew characters, although under these circumstances, one needs to verify that $\chi^{\lambda / \nu}=0$ unless $\lambda / \nu$ is a legitimate skew partition (i.e., $D_{\lambda} \supseteq D_{\nu}$ ). Further details regarding the skew characters of $S_{n}$ can be found in [GW, JK, M1].

LEMMA 3.4. If $\lambda / \nu$ is a skew partition of $n$ and $w \in S_{n}$ is an $n$-cycle, then

$$
I_{\lambda / \nu}(q)=\chi^{\lambda / \nu}\left(w^{r}\right) \quad \text { at } q=e^{2 \pi i r / n} \quad(0 \leq r<n) .
$$

Proof. Let $c(\lambda, \mu, \nu)$ denote the multiplicity of $\chi^{\mu}$ in $\chi^{\lambda / \nu}$ (the Littlewood-Richardson coefficient), so that

$$
\chi^{\lambda / \nu}=\sum_{|\mu|=n} c(\lambda, \mu, \nu) \chi^{\mu} .
$$

It is well-known [M1] that the multiplicities $c(\lambda, \mu, \nu)$ also describe the Schur function expansion of $s_{\lambda / \nu}$; i.e.,

$$
s_{\lambda / \nu}\left(x_{1}, x_{2}, \ldots\right)=\sum_{|\mu|=n} c(\lambda, \mu, \nu) s_{\mu}\left(x_{1}, x_{2}, \ldots\right) .
$$

By Lemma 3.1, it therefore suffices to restrict our attention to the case $\nu=\varnothing$.

Since any $n$-cycle $w$ (acting on $\mathbf{C}^{n}$ via permutation of the natural coordinates) is $e^{2 \pi i / n}$-regular, Theorems 2.2 and 3.2 immediately imply $I_{\lambda}(q)=P_{\lambda, w}(q) \bmod 1-q^{n}$. Therefore,

$$
I_{\lambda}\left(e^{2 \pi i r / n}\right)=P_{\lambda, w}\left(e^{2 \pi i r / n}\right)=\chi^{\lambda}\left(w^{r}\right) .
$$


REMARK. Although Lemma 3.4 is essentially a corollary of Theorems 2.2 and 3.2, it is also possible to give an elementary proof which does not require the invariant theory of reflection groups. To construct such a proof, let $\omega=e^{2 \pi i / n}$, and fix $r(0 \leq r<n)$. Let $\mu$ denote the cycle-type of $w^{r}$ ( $w=n$-cycle), and let $z_{\mu}$ denote the size of the $S_{n}$-centralizer of $w^{r}$. As a first step, show that

$$
\lim _{q \rightarrow \omega^{r}}(q)_{n} p_{\nu}\left(1, q, q^{2}, \ldots\right)=\delta_{\mu \nu} z_{\mu}
$$

which is a simple calculation. Next, use the identity [M1, (7.10)]

$$
s_{\lambda}\left(x_{1}, x_{2}, \ldots\right)=\sum_{|\nu|=n} \frac{1}{z_{\nu}} \chi^{\lambda}(\nu) p_{\nu}\left(x_{1}, x_{2}, \ldots\right)
$$

to conclude that

$$
(q)_{n} s_{\lambda}\left(1, q, q^{2}, \ldots\right)=\chi^{\lambda}\left(w^{r}\right) \text { at } q=\omega^{r} .
$$

Lemma 3.4 may now be deduced from Lemma 3.1.

Proof of Theorem 3.3. Let $l=\ell(\mu)$ and let $T$ be a standard tableau of shape $\lambda$. Let $\lambda^{j}$ denote the shape of the diagram formed by the cells of $D_{\lambda}$ labeled $\leq \mu_{1}+\cdots+\mu_{j}$ by $T$. (In particular, $\lambda^{0}=\varnothing$.) We refer to the partition sequence $\left(\lambda^{j}: 0 \leq j \leq l\right)$ as a $\mu$-partition of $\lambda$ since $\left|\lambda^{j}\right|-\left|\lambda^{j-1}\right|=\mu_{j}$ and $D_{\lambda^{0}} \subseteq D_{\lambda^{1}} \subseteq \cdots D_{\lambda^{l}}=D_{\lambda}$.

The presence of $\mu_{1}+\cdots+\mu_{j} \in D(T)$ will contribute $m$ to $\operatorname{ind}_{\mu}(T)$, and so does not affect ind $\operatorname{in}_{\mu}(T) \bmod m$. We therefore have

$$
\operatorname{ind}_{\mu}(T)=\sum_{j} \frac{m}{\mu_{j}} \text { ind } T_{j} \quad(\bmod m),
$$

where $T_{j}$ denotes the restriction of $T$ to $D_{\lambda^{j} / \lambda^{j-1}}$, renumbered from 1 to $\mu_{j}$, so that $T_{j}$ is a legitimate standard tableau. It follows that

$$
\sum_{T \in \mathscr{F}^{\lambda}} q^{\operatorname{ind}_{\mu}(T)}=\sum_{\left(\lambda^{\prime}\right)} I_{\lambda^{1} / \lambda^{0}}\left(q^{m / \mu_{1}}\right) \cdots I_{\lambda^{l} / \lambda^{l-1}}\left(q^{m / \mu_{l}}\right) \quad\left(\bmod 1-q^{m}\right),
$$

summed over all $\mu$-partitions $\left(\lambda^{j}: 0 \leq j \leq l\right)$ of $\lambda$.

Now consider the character values $\chi^{\lambda}\left(w^{r}\right)$, where $w$ is of cycletype $\mu$. Let $w=w_{1} \cdots w_{l}$ be the factorization of $w$ into commuting cycles of lengths $\mu_{1}, \ldots, \mu_{l}$, and regard $w$ as an element of the Young subgroup $S_{\mu_{1}} \times \cdots \times S_{\mu_{l}}$. By repeated application of (3.4), it follows that

$$
\chi^{\lambda}\left(w^{r}\right)=\sum_{(\lambda)} \chi^{\lambda^{1} / \lambda^{0}}\left(w_{1}^{r}\right) \cdots \chi^{\lambda^{l} / \lambda^{l-1}}\left(w_{l}^{r}\right),
$$

summed over all $\mu$-partitions $\left(\lambda^{j}: 0 \leq j \leq l\right)$ of $\lambda$. 
At $q=\omega^{r}\left(\omega=e^{2 \pi i / m}\right)$, we have $q^{m / \mu_{\jmath}}=e^{2 \pi i r / \mu_{\jmath}}$, so Lemma 3.4 shows that the terms appearing in (3.6) and (3.7) are identical. Thus,

$$
\sum_{T \in \mathscr{F}^{\lambda}} q^{\operatorname{ind}_{\mu}(T)}=\chi^{\lambda}\left(w^{r}\right) \quad \text { at } q=\omega^{r}
$$

Apply Proposition 1.1 to complete the proof.

4. Wreath products. Let $G$ be a finite group. In this section, we will show that the cyclic exponents of the wreath product $G$ ? $S_{n}$ can be described combinatorially in terms of the cyclic exponents of $G$. We first give a brief survey (Part A) on the representations of wreath products, and we give a recurrence for the irreducible characters analogous to the Murnaghan-Nakayama rule. Since this recurrence appears to be new, we have included a proof in the Appendix ( $\S 7)$. In Part B, we construct some statistical parameters based on the cyclic exponents of $G$. These parameters will be used to describe the cyclic exponents of $G$ ? $S_{n}$ in Part C. For further details on the representation theory of wreath products, the reader is referred to [JK, K, M2].

A. Background. Use $G_{n}$ as an abbreviation for $G i S_{n}$. (In particular, $G_{0}$ is the group with one element.) We may identify $G_{n}$ informally as the group of $n \times n$ pseudo permutation matrices in which the nonzero entries are chosen from $G$. Note that $S_{n}$ is a natural homomorphic image of $G_{n}$; references to the cycle structure of any $x \in G_{n}$ should thus be interpreted with respect to the underlying $S_{n}$-image of $x$.

Suppose that $\left(i_{1}, \ldots, i_{k}\right)$ is a cycle in (the $S_{n}$-image of) some $x \in$ $G_{n}$. If $g_{1}, \ldots, g_{k} \in G$ are the corresponding nonzero entries in rows $i_{1}, \ldots, i_{k}$ of $x$, then the cycle-product $g_{k} \cdots g_{2} g_{1}$ is well-defined up to $G$-conjugacy (i.e., cyclic shifts of $i_{1}, \ldots, i_{k}$ will not affect the conjugacy class of the cycle product). We refer to this conjugacy class as the $G$ class of the cycle. It will sometimes be preferable to abuse this notation and label the $G$-class of a cycle with any convenient element $g$ in the class.

Choose a fixed $x \in G_{n}$. For each conjugacy class $c \in C_{G}$, let $\mu^{c}$ denote the partition formed by the lengths of the cycles of $x$ with $G$-class $c$. We may thus associate with $x$ a partition-valued function $\underline{\mu}: C_{G} \rightarrow \mathscr{P}$ with $|\underline{\mu}|=n$, where

$$
|\underline{\mu}|=\sum_{c \in C_{G}}\left|\mu^{c}\right|
$$


We will refer to $\mu$ as the type of $x$. Two elements of $G_{n}$ are conjugate iff they have the same type (cf. [M2, §9]), and so the conjugacy classes of $G_{n}$ are indexed by the partition-valued functions $\mu$.

Let $U$ be a $G$-module and let $V$ be an $S_{n}$-module. The wreath product $U \imath V$ is the $G_{n}$-module whose vector-space structure is $\left(U^{\otimes n}\right) \otimes V$, and whose module structure may be described as follows. If $\left(g_{1}, \ldots, g_{n}\right) \in G^{n}$ (the subgroup of diagonal matrices), then

$$
\left(g_{1}, \ldots, g_{n}\right) \circ\left(u_{1} \otimes \cdots \otimes u_{n} \otimes v\right)=g_{1} u_{1} \otimes \cdots \otimes g_{n} u_{n} \otimes v,
$$

and if $w \in S_{n}$ (the subgroup of permutation matrices), then

$$
w \circ\left(u_{1} \otimes \cdots \otimes u_{n} \otimes v\right)=u_{w^{-1}(1)} \otimes \cdots \otimes u_{w^{-1}(n)} \otimes w v
$$

for all $u_{i} \in U, v \in V$. Since $G_{n}=G^{n} \rtimes S_{n}$ (semidirect product), this completely defines the $G_{n}$-module structure of $U ? V$.

It is not hard to show that $U ? V$ is irreducible if both $U$ and $V$ are irreducible; however, not all irreducible representations of $G_{n}$ arise in this fashion. To obtain a complete set of irreducible representations (a problem originally solved by Specht [Spe]), one needs to consider representations induced from wreath analogues of Young subgroups.

Given nonnegative integers $n_{1}, \ldots, n_{t}$ such that $n_{1}+\cdots+n_{t}=n$, let $S_{(n)}$ denote any Young subgroup of $S_{n}$ isomorphic to $S_{n_{1}} \times \cdots \times S_{n_{t}}$, and let $G_{(n)}=G ? S_{(n)}$ denote the corresponding subgroup of $G_{n}$.

THEOREM 4.1 (Specht). Let $U_{1}, \ldots, U_{t}$ be a complete list of irreducible, inequivalent $G$-modules. A complete list of irreducible, inequivalent $G$ ? $S_{n}$-modules consist of the representations

$$
\left(U_{1} \prec V_{1}\right) \otimes \cdots \otimes\left(U_{t} \prec V_{t}\right) \uparrow G_{n}
$$

induced from the subgroups $G_{(n)}$, where $n_{1}, \ldots, n_{t}$. range over all nonnegative integers with $n_{1}+\cdots+n_{t}=n$ and $V_{i}$ ranges over a list of the irreducible, inequivalent $S_{n_{1}}$-modules.

We remark that in case $n_{i}=0$, the factor $U_{i} 2 V_{i}$ should be interpreted as a trivial one-dimensional module.

Proofs of Specht's theorem can be found in [JK, K, M2, Spe].

In place of the indices $i=1, \ldots, t$ used in Theorem 4.1, we will sometimes use the irreducible characters $I_{G}$. In particular, if $\theta=$ char $U_{i}$, we will write $n_{\theta}=n_{i}$ and $V_{\theta}=V_{i}$. In these terms, observe that (4.1) may be labeled by a partition-valued function $\underline{\lambda}: I_{G} \rightarrow \mathscr{P}$, where $\lambda^{\theta}$ is the partition of $n_{\theta}$ corresponding to the $S_{n_{\theta}}$-module $V_{\theta}$. 


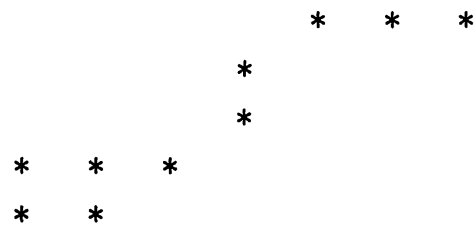

FIGURE 3(a)

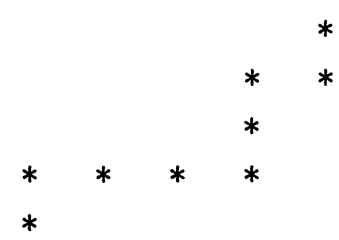

Figure 3(b)

Specht's theorem thus yields a natural bijection between the irreducible $G_{n}$-modules and the functions $\underline{\lambda}: I_{G} \rightarrow \mathscr{P}$ with $|\underline{\lambda}|=n$, where

$$
|\underline{\lambda}|=\sum_{\theta \in I_{G}}\left|\lambda^{\theta}\right| \text {. }
$$

In the following, it will be convenient to associate with $\underline{\lambda}$ a skew partition of $n$ obtained by translating the various component diagrams $D_{\lambda^{\theta}}: \theta \in I_{G}$ in the plane $\mathbf{Z}^{2}$ so that the rows and columns occupied by the $D_{\lambda \theta}$ 's are disjoint. The actual translations used are unimportant; we only care that the resulting arrangement of cells forms a legitimate skew diagram. For example, an appropriate arrangement for the partitions $(3,2),(1,1)$ and (3) appears in Figure 3(a). By abuse of notation, we will refer to any such arrangement as the diagram of $\underline{\lambda}$, and let $\mathscr{F} \underline{\lambda}$ denote the collection of standard tableaux of this shape.

Let $\chi^{\underline{\lambda}}$ denote the irreducible $G_{n}$-character indexed by $\underline{\lambda}$. A further consequence of Specht's theorem is the following.

Corollary 4.2 (cf. [M2, p. 200]). If $\left|\lambda^{\theta}\right|=n_{\theta}$, then $\operatorname{deg} \chi^{\underline{\lambda}}=$ $\mid \mathscr{F}$ 기 $\mid \cdot \prod_{\theta \in I_{G}}(\operatorname{deg} \theta)^{n_{\theta}}$.

Proof. The subgroup $G_{(n)}$ is of index $n ! / \prod_{\theta} n_{\theta}$ ! in $G_{n}$. It follows that the representation indexed by $\underline{\lambda}$ (cf. (4.1)) is of degree

$$
n ! \prod_{\theta} \frac{(\operatorname{deg} \theta)^{n_{\theta}}}{n_{\theta} !} \cdot\left|\mathscr{F}^{\lambda^{\theta}}\right| .
$$

The result now follows from the fact that there is a simple bijection (left as an exercise) which proves

$$
\left|\mathscr{F}^{\lambda}\right|=n ! \prod_{\theta} \frac{1}{n_{\theta} !}\left|\mathscr{F}^{\lambda^{\theta}}\right|
$$

Let $\mu, \lambda: I_{G} \rightarrow \mathscr{P}$ be a pair of partition-valued functions with $|\lambda|-$ $|\underline{\mu}|=\bar{n}$ and $\underline{\lambda} \supseteq \underline{\mu}$ (i.e., $D_{\lambda^{\theta}} \supseteq D_{\mu^{\theta}}$ for all $\theta \in I_{G}$ ). The collection of skew partitions $\underline{\lambda} / \underline{\mu}=\left\{\lambda^{\theta} / \mu^{\theta}: \theta \in I_{G}\right\}$ may be used to index a 
skew representation of $G_{n}$; namely, the $G_{n}$-module of the form (4.1) in which $V_{\theta}$ is the skew $S_{n_{\theta}}$-module indexed by $\lambda^{\theta} / \mu^{\theta}\left(n_{\theta}=\left|\lambda^{\theta}\right|-\left|\mu^{\theta}\right|\right)$. Let $\chi^{\lambda}-\underline{\mu}$ denote the corresponding $G_{n}$-character.

The Murnaghan-Nakayama rule is a combinatorial recurrence for evaluating the $S_{n}$-characters $\chi^{\lambda}$ as well as the skew characters $\chi^{\lambda / \mu}[\mathbf{J K}$, 2.4]. To analyze the cyclic exponents of $G \succ S_{n}$, we will need a similar recurrence for the irreducible characters $\chi^{\lambda}$ as well as the skew characters $\chi^{\lambda /} \underline{\mu}$. To describe this recurrence, we first recall that a skew hook (also known as a rim hook [JK] or border strip [M1]) is a rookwise connected skew partition with no $2 \times 2$ square as a subdiagram. An example appears in Figure 3(b). More generally, we define a skew family $\lambda / \mu$ to be a a skew hook if only one of the components $\lambda^{\theta} / \mu^{\theta}$ is nonempty and this one component is a skew hook. The character of a skew hook $\lambda / \mu$ is defined to be the unique $\theta \in I_{G}$ for which $\lambda^{\theta} \neq \mu^{\theta}$. A partition of $\underline{\lambda} / \underline{\mu}$ into skew hooks is a nested sequence

$$
\underline{\mu}=\underline{\lambda}^{0} \subseteq \underline{\lambda}^{1} \subseteq \cdots \subseteq \underline{\lambda^{l}}=\underline{\lambda}
$$

of partition-valued functions $\underline{\lambda}^{i}: I_{G} \rightarrow \mathscr{P}$ such that each of $\underline{\lambda}^{i} / \underline{\lambda}^{i-1}$ is a skew hook $(1 \leq i \leq l)$. If $\left|\underline{\lambda}^{i}\right|-\left|\underline{\lambda}^{i-1}\right|=\beta_{i}$, then (4.2) is said to be a $\beta$-partition.

THeOREM 4.3. Assume that the cycles of $x \in G_{n}$ are of length $\beta_{1}, \ldots$, $\beta_{l}$ and that the corresponding $G$-classes are $g_{1}, \ldots, g_{l}$. We have

$$
\chi^{\lambda}-\underline{\mu}(x)=\sum_{\left(\underline{\lambda}^{\prime}\right)} \prod_{i=1}^{l}(-1)^{r_{i}-1} \theta_{i}\left(g_{i}\right),
$$

summed over all $\beta$-partitions $\left(\underline{\lambda}^{i}\right)$ of $\underline{\lambda} \underline{\mu}$ into skew hooks, where $\theta_{i}$ denotes the character of $\underline{\lambda}^{i} / \underline{\lambda}^{i-1}$ and $r_{i}$ denotes the number of nonempty rows in the $\theta_{i}$ th component of $\underline{\lambda}^{i} / \underline{\lambda}^{i-1}$.

The proof is given in $\S 7 \mathrm{~A}$.

B. Parameters for the cyclic exponents of $G$. For each $\theta \in I_{G}$, choose a totally ordered alphabet $A_{\theta}$ of size $\operatorname{deg} \theta$. Using $A_{\theta}$ as an index set, let $\left\{e_{g}(a): a \in A_{\theta}\right\}$ denote the cyclic exponents of $g \in G$ with respect to $\theta$. Following the conventions of $\S 1$, let

$$
P_{\theta, g}(q)=\sum_{a \in A_{\theta}} q^{e_{g}(a)}
$$

denote the associated generating function. 
Indexing exponents with the letters of $A_{\theta}$ induces a natural weighting on words over $A_{\theta}$. Specifically, we define the $g$-weight of any word $\alpha=a_{1} a_{2} \cdots a_{m} \in A_{\theta}^{m}$ via

$$
|\alpha|_{g}=\sum_{i=1}^{l} e_{g}\left(a_{i}\right)
$$

Observe that the cyclic group $C_{m}$ acts on $A_{\theta}^{m}$ via cyclic shifts. Let fix $\alpha$ denote the order of the stabilizer of any $\alpha \in A_{\theta}^{m}$. If $f=$ fix $\alpha$, then the $C_{m}$-orbit of $\alpha$ consists of $m / f$ distinct words; among these words there must be a unique word that is lexicographically smallest with respect to the ordering on $A_{\theta}$. Using $\sigma$ to denote the basic shift operation

$$
a_{1} a_{2} \cdots a_{m} \stackrel{\sigma}{\longmapsto} a_{2} \cdots a_{m} a_{1},
$$

we define the phase $\phi(\alpha)$ to be the integer $i(0 \leq i<m / f)$ for which $\sigma^{i} \alpha$ achieves this lexicographic minimum. For example, if $\alpha=a c a b a c a b a c a b$ and $a<b<c$, then fix $\alpha=3$ and $\phi(\alpha)=2$.

More generally, if $C_{n}$ is a subgroup of $C_{m}$ (and hence, $n \mid m$ ), we may interpret $(n, \mathrm{fix} \alpha)$ as the order of the $C_{n}$-stabilizer of $\alpha$, and we define the $n$-phase $\phi_{n}(\alpha)$ as above, but with respect to the $C_{n}$-orbit of $\alpha \in A_{\theta}^{m}$. Thus, $\phi_{n}(\alpha)$ is the integer $i(0 \leq i<n /(n$, fix $\alpha))$ for which $\sigma^{i m / n} \alpha$ is the lexicographically smallest member of the $C_{n}$-orbit of $\alpha$. It will also be convenient to interpret $(n, \mathrm{fix} \alpha)$ and extend the notation $\phi_{n}(\alpha)$ to situations in which $n \nmid m$. In such cases, we note that $(n$, fix $\alpha)$ is the order of the $C_{(n, m)}$-stabilizer of $\alpha$, and we will use $\phi_{n}(\alpha)$ as an abbreviation for the $(n, m)$-phase of $\alpha$.

If $g \in G$ is of order $k$ and $n$ is a positive integer, we define the cyclic weight of $\alpha \in A_{\theta}^{m}$ with respect to $g$ and $n$ via

and let

$$
\operatorname{cyc}_{g}^{(n)}(\alpha)=|\alpha|_{g}+k(n, \text { fix } \alpha) \phi_{n}(\alpha),
$$

$$
C_{\theta, g, m}^{(n)}(q)=\sum_{\alpha \in A_{\theta}^{m}} q^{\mathrm{cyc}_{g}^{(n)}(\alpha)}
$$

denote the associated generating function. We remark that if $\operatorname{deg} \theta=$ 1 , then there is a single exponent $e_{g}$ for each $g \in G$, and we have $C_{\theta, g, m}^{(n)}(q)=q^{m e_{g}}=P_{\theta, g}(q)^{m}$.

LEMMA 4.4. If $g \in G$ is of order $k$ and $\theta \in I_{G}$, then

$$
C_{\theta, g, m}^{(n)}(q)=\sum_{s \mid(m, n)} P_{\theta, g}\left(q^{s}\right)^{m / s} \sum_{r \mid s} \mu\left(\frac{s}{r}\right) \frac{r}{(m, n)} \frac{1-q^{k(m, n)}}{1-q^{k r}},
$$

where $\mu$ denotes the classical Möbius function. 
Proof. Let $d=(m, n)$. For any $A \subseteq A_{\theta}^{m}$, let $\mathrm{GF}[A]$ denote the generating function for the $g$-weights in $A$; i.e.,

$$
\mathrm{GF}[A]=\sum_{\alpha \in A} q^{|\alpha|_{g}}
$$

Note that for any $s \mid m$, we have

$$
P_{\theta, g}\left(q^{s}\right)^{m / s}=\mathrm{GF}\left[\alpha \in A_{\theta}^{m}: s \mid \text { fix } \alpha\right] .
$$

If $s \mid d$, then we have $s \mid(d$, fix $\alpha)$ iff $s \mid$ fix $\alpha$, and hence

$$
\mathrm{GF}\left[\alpha \in A_{\theta}^{m}: s \mid \mathrm{fix} \alpha\right]=\sum_{s|r| d} \mathrm{GF}\left[\alpha \in A_{\theta}^{m}: r=(d, \mathrm{fix} \alpha)\right] \text {. }
$$

An application of Möbius inversion therefore yields

$$
\mathrm{GF}\left[\alpha \in A_{\theta}^{m}: r=(d, \mathrm{fix} \alpha)\right]=\sum_{r|s| d} \mu\left(\frac{s}{r}\right) P_{\theta, g}\left(q^{s}\right)^{m / s} .
$$

If $r=(d$, fix $\alpha)$ then there are $d / r$ words in the $C_{d}$-orbit of $\alpha$, and these words all have the same $g$-weight. Consequently, the cyclic weights of these words are of the form $|\alpha|_{g}+k r i(0 \leq i<d / r)$, and we have

$$
\begin{aligned}
\sum_{\alpha \in A_{\theta}^{m}} q^{\mathrm{cyc}_{g}^{(n)}(\alpha)} & =\sum_{r \mid d} \frac{r}{d} \frac{1-q^{k d}}{1-q^{k r}} \operatorname{GF}\left[\alpha \in A_{\theta}^{m}: r=(d, \text { fix } \alpha)\right] \\
& =\sum_{r|s| d} \mu\left(\frac{s}{r}\right) \frac{r}{d} \frac{1-q^{k d}}{1-q^{k r}} P_{\theta, g}\left(q^{s}\right)^{m / s}
\end{aligned}
$$

LEMMA 4.5. Let $g, k, \theta$ be as above, and let $\omega=e^{2 \pi i / k n}$. Let $l=$ $n /(n, j)$ denote the order of $\omega^{j k}$. If $l \mid m$, then

$$
C_{\theta, g, m}^{(n)}\left(\omega^{j}\right)=P_{\theta, g}\left(\omega^{[n, j]}\right)^{m / l}=\theta\left(g^{j /(n, j)}\right)^{m / l} .
$$

Proof. Let $d=(m, n)$. We have $l \mid m$ and $l \mid n$, so $1-q^{k d}=0$ at $q=\omega^{j}$. Since $1-q^{k r}=0$ at $q=\omega^{j}$ iff $l \mid r$, it follows that

$$
\lim _{q \rightarrow \omega^{\prime}}=\frac{r}{d} \frac{1-q^{k d}}{1-q^{k r}}= \begin{cases}1 & \text { if } l \mid r \\ 0 & \text { otherwise. }\end{cases}
$$

Hence, Lemma 4.4 implies

$$
C_{\theta, g, m}^{(n)}\left(\omega^{j}\right)=\sum_{l|r| s \mid d} \mu\left(\frac{s}{r}\right) P_{\theta, g}\left(\omega^{j s}\right)^{m / s} .
$$


However,

$$
\sum_{l|r| s} \mu\left(\frac{s}{r}\right)= \begin{cases}1 & \text { if } l=s \\ 0 & \text { otherwise }\end{cases}
$$

so we have

$$
C_{\theta, g, m}^{(n)}\left(\omega^{j}\right)=P_{\theta, g}\left(\omega^{j l}\right)^{m / l}=P_{\theta, g}\left(\omega^{[n, j]}\right)^{m / l} .
$$

C. The cyclic exponents of $G$ ? $S_{n}$. Choose a fixed irreducible character $\chi^{\lambda}$ of $G_{n}$. Extending the notation of $\S 3 \mathrm{~A}$, let $I_{\underline{\lambda}}(q)$ denote the generating function for the indices of the standard tableaux of shape $\underline{\lambda}$. Let $P_{\underline{\lambda}, x}(q)$ denote the usual generating function for the cyclic exponents of $x \in G_{n}$ with respect to $\chi \lambda$. We first consider $n$-cycles.

THEOREM 4.6. Let $n_{\theta}=\left|\lambda^{\theta}\right|$. If $x \in G_{n}$ is an $n$-cycle whose $G$-class $g$ is of order $k$, then

$$
P_{\underline{\lambda}, x}(q)=I_{\underline{\lambda}}\left(q^{k}\right) \prod_{\theta \in I_{G}} C_{\theta, g, n_{\theta}}^{(n)}(q) \quad\left(\bmod 1-q^{k n}\right) .
$$

Remark. Using the constructions in Part $\mathrm{B}$, it is easy to give a combinatorial description of the cyclic exponents of $x$. Specifically, they are of the form

$$
k \cdot \operatorname{ind} T+\sum_{\theta} \operatorname{cyc}_{g}^{(n)}\left(\alpha_{\theta}\right) \quad(\bmod k n),
$$

where $T$ varies over the standard tableaux of shape $\underline{\lambda}$, and $\alpha_{\theta}$ varies over the $A_{\theta}$-words of length $n_{\theta}$.

Proof. The diagram of $\underline{\lambda}$ indexes both an irreducible character of $G_{n}$ and a skew character of $S_{n}$. In order to avoid ambiguity, we will denote the latter character by $\chi_{S}^{\frac{\lambda}{S}}$.

The cycles of $x^{j}$ all have the same length $l=n /(n, j)$ and the same $G$-class, namely $g^{j /(n, j)}$. To partition a component $\lambda^{\theta}$ of $\underline{\lambda}$ into skew hooks of size $l$ certainly requires $l \mid n_{\theta}$. Therefore, if $w$ is the $S_{n}$-image of $x$, the character recurrences for $G_{n}$ and $S_{n}$ (Theorem 4.3) imply

$$
\chi^{\lambda}\left(x^{j}\right)=\chi \frac{\lambda}{S}\left(w^{j}\right)=0
$$

unless $l \mid n_{\theta}$ for all $\theta \in I_{G}$. Assuming this necessary condition is satisfied, then we have

$$
\chi^{\underline{\lambda}}\left(x^{j}\right)=\chi_{S}^{\frac{\lambda}{S}}\left(w^{j}\right) \prod_{\theta} \theta\left(g^{j /(n, j)}\right)^{n_{\theta} / l},
$$


since $n_{\theta} / l$ counts the number of times a skew hook of size $l$ must be removed from the component $\lambda^{\theta}$.

Now if $\omega=e^{2 \pi i / k n}$ and $l \mid n_{\theta}$, then Lemma 4.5 implies

$$
C_{\theta, g, n_{\theta}}^{(n)}\left(\omega^{j}\right)=\theta\left(g^{j /(n, j)}\right)^{n_{\theta} / l}
$$

and Lemma 3.4 implies

$$
I_{\underline{\lambda}}\left(\omega^{k j}\right)=\chi \frac{\lambda}{S}\left(w^{j}\right)
$$

so it follows that

$$
\chi^{\underline{\lambda}}\left(x^{j}\right)=I_{\underline{\lambda}}\left(\omega^{k j}\right) \prod_{\theta} C_{\theta, g, n_{\theta}}^{(n)}\left(\omega^{j}\right) \quad(0 \leq j<k n) .
$$

Apply Proposition 1.1.

We remark that Theorem 4.6 generalizes easily to the skew representations of $G_{n}$; the same proof holds verbatim.

Now consider the cyclic exponents of an arbitrary element $x \in G_{n}$. Let $x=x_{1} \ldots x_{l}$ be a factorization of $x$ into disjoint commuting cycles. Assume that $x_{i}$ is a cycle of length $\mu_{i}$ whose $G$-class $g_{i}$ is of order $k_{i}$ $(1 \leq i \leq l)$. Let $M=\left[k_{1} \mu_{1}, \ldots, k_{l} \mu_{l}\right]$ denote the order of $x$ and let $m=\left[\mu_{1}, \ldots, \mu_{l}\right]$ denote the order of the $S_{n}$-image of $x$.

If $T$ is a standard tableau of shape $\underline{\lambda}$, let $\underline{\lambda}^{i}=\underline{\lambda}^{i}(T)$ denote the shape of the diagram formed by the cells of $\underline{\lambda}$ numbered $\leq \mu_{1}+\cdots+\mu_{i}$ by $T$. We have

$$
\varnothing=\underline{\lambda}^{0} \subseteq \underline{\lambda}^{1} \subseteq \cdots \subseteq \underline{\lambda}^{l}=\underline{\lambda}
$$

and $\left|\underline{\lambda}^{i}\right|-\left|\underline{\lambda}^{i-1}\right|=\mu_{i}$, so the sequence $\left(\underline{\lambda}^{i}: 0 \leq i \leq l\right)$ forms a $\mu$-partition of $\underline{\lambda}$, as in (4.2). Given such a partition, define

$$
n_{i, \theta}(T)=n_{i, \theta}=\left|\lambda^{\theta} \cap\left(\underline{\lambda}^{i} / \underline{\lambda}^{i-1}\right)\right|,
$$

and observe that $\sum_{i} n_{i, \theta}=n_{\theta}, \sum_{\theta} n_{i, \theta}=\mu_{i}$.

THEOREM 4.7. In terms of the above notation, the cyclic exponents of $x$ with respect to $\chi^{\lambda}$ are of the form

$$
\frac{M}{m} \operatorname{ind}_{\mu}(T)+\sum_{i, \theta} \frac{M}{k_{i} \mu_{i}} \operatorname{cyc}_{g_{i}}^{\left(\mu_{i}\right)}\left(\alpha_{i, \theta}\right) \quad(\bmod M),
$$

where $T$ ranges over the standard tableaux of shape $\underline{\lambda}$ and $\alpha_{i, \theta}$ varies over the $A_{\theta}$-words of length $n_{i, \theta}(T)$.

Proof. Regard $x$ as a member of the "Young" subgroup $G_{(\mu)}$ (cf. the paragraph preceding Theorem 4.1). By repeated application of 
Lemma 7.1, we have

$$
\chi^{\underline{\lambda}}\left(x^{j}\right)=\sum_{\left(\underline{\lambda}^{i}\right)} \chi^{\underline{\lambda}^{1} / \underline{\lambda}^{0}}\left(x_{1}^{j}\right) \cdots \chi^{\underline{\lambda}^{\prime} / \underline{\lambda}^{l-1}}\left(x_{l}^{j}\right),
$$

summed over all $\mu$-partitions $\left(\underline{\lambda}^{i}: 0 \leq i \leq l\right)$ of $\underline{\lambda}$.

Let $\omega=e^{2 \pi i / M}$. Since $P_{\chi, y}\left(\omega^{M j / k}\right)=\chi\left(y^{j}\right)$ for any character $\chi$ and any $y$ of order $k$, Proposition 1.1 therefore implies

$$
P_{\underline{\lambda}, x}(q)=\sum_{\left(\underline{\lambda}^{i}\right)} P_{\underline{\lambda}^{1} / \underline{\lambda}^{0}, x_{l}}\left(q^{M / k_{1} \mu_{1}}\right) \cdots P_{\underline{\lambda}^{l} / \underline{\lambda}^{l-1}, x_{l}}\left(q^{M / k_{l} \mu_{l}}\right) \quad\left(\bmod 1-q^{M}\right) .
$$

Hence, from Theorem 4.6 we may deduce

$$
P_{\underline{\lambda}, x}(q)=\sum_{\left(\underline{\lambda}^{i}\right)} \prod_{i=1}^{l} I_{\underline{\lambda}^{i} / \underline{\lambda}^{i-1}}\left(q^{M / \mu_{l}}\right) \prod_{\theta} C_{\theta, g_{t}, n_{l, \theta}}^{\left(\mu_{i}\right)}\left(q^{M / k_{i} \mu_{t}}\right) \quad\left(\bmod 1-q^{M}\right) .
$$

In view of (3.6),

$$
\prod_{i=1}^{l} I_{\underline{\lambda}^{i} / \underline{\lambda}^{i-1}}\left(q^{M / \mu_{i}}\right)
$$

may be identified $\left(\bmod 1-q^{M}\right)$ as the generating function for $\frac{M}{m} \operatorname{ind}_{\mu}(T)$, where $T$ ranges over the standard tableaux of shape $\underline{\lambda}$ which produce the $\mu$-partition $\left(\underline{\lambda}^{i}: 0 \leq i \leq l\right)$. To complete the proof, recall (cf. (4.3)) that $C_{\theta, g_{l}, n_{l, \theta}}^{\left(\mu_{t}\right)}\left(q^{M / k_{i} \mu_{\imath}}\right)$ is the generating function for

$$
\frac{M}{k_{i} \mu_{i}} \operatorname{cyc}_{g_{i}}^{\left(\mu_{i}\right)}(\alpha)
$$

where $\alpha$ varies over the $A_{\theta}$-words of length $n_{i, \theta}(T)$.

5. The reflection groups $C_{m} \geq S_{n}$. Let $C_{m}$ denote the cyclic group of order $m$. In this section we will illustrate the results of the previous sections for the reflection group $C_{m} 2 S_{n}$ (sometimes known as the generalized symmetric group). We will find that the description of the cyclic exponents simplifies considerably from the general setting of $\S 4$. Also, we will derive explicit formulas and combinatorial interpretations for the generalized exponents; this will provide an opportunity to illustrate a particular case of Springer's result (Theorem 2.2).

A. Cyclic exponents. Let $\theta$ be an irreducible $C_{m}$-character. Since $\operatorname{deg} \theta=1$, there is only one word of each length over the alphabet $A_{\theta}$ of $\S 4 \mathrm{~B}$; the phase of such a word is clearly zero. Consequently, if $e_{g}(\theta)$ is the cyclic exponent of $g \in C_{m}$ with respect to $\theta$, then we have (cf. (4.3))

$$
C_{\theta, g, k}^{(n)}(q)=q^{k e_{g}(\theta)} \text {. }
$$


Regard $C_{m}$ as the group of $m$ th roots of unity, let $\omega=e^{2 \pi i / m}$, and let $\varphi^{k}$ denote the irreducible $C_{m}$-character defined by $\varphi^{k}(\omega)=\omega^{k}$. The cyclic exponent of $\omega^{j}$ with respect to $\varphi^{k}$ is clearly $j k(\bmod m)$.

In the following it will be convenient to index the irreducible characters of $C_{m}\left\{S_{n}\right.$ by the partition-valued functions $\underline{\lambda}: I_{m} \rightarrow \mathscr{P}$ with $|\underline{\lambda}|=n$, where the index set $I_{m}:=\{0,1, \ldots, m-1\}$ is used in place of $\left\{\varphi^{0}, \ldots, \varphi^{m-1}\right\}$. Under this convention, we define

$$
r(\underline{\lambda})=\sum_{i=0}^{m-1} i\left|\underline{\lambda}^{i}\right|
$$

and we leave to the reader the easy task of verifying that the following result is a direct corollary of Theorem 4.6.

THEOREM 5.1. If $x \in C_{m}$ i $S_{n}$ is an n-cycle of $C_{m}$-class $\omega$, then

$$
P_{\underline{\lambda}, x}(q)=q^{r(\underline{\lambda})} I_{\underline{\lambda}}\left(q^{m}\right) \quad\left(\bmod 1-q^{m n}\right) .
$$

In particular, the cyclic exponents of $x$ with respect to $\underline{\lambda}$ are of the form

$$
r(\underline{\lambda})+m \cdot \operatorname{ind} T(\bmod m n)
$$

where $T$ varies over the standard tableaux of shape $\underline{\lambda}$.

For the general case, assume $x \in C_{m} 2 S_{n}$ has cycle lengths $\mu_{1}, \ldots, \mu_{l}$ and corresponding $C_{m}$-classes $\omega^{e_{1}}, \ldots, \omega^{e_{l}}$. Let $k_{i}=m /\left(m, e_{i}\right)$ denote the order of $\omega^{e_{i}}$, let $M$ denote the order of $x$, and let $m_{\mu}$ denote the order of the $S_{n}$-image of $x$. (To indicate the latter by " $m$ " would produce unfortunate confusion here.) For each standard tableau $T$ of shape $\underline{\lambda}$, let $\underline{\lambda}^{i}=\underline{\lambda}^{i}(T): I_{m} \rightarrow \mathscr{P}(0 \leq j \leq l)$ denote the $\mu$-partition of $\underline{\lambda}$ defined by $T$, as described in $\S 4 \mathrm{C}$. As a corollary of Theorem 4.7, we have

THEOREM 5.2. The cyclic exponents of $x$ with respect to $\underline{\lambda}$ are

$$
\frac{M}{m_{\mu}} \operatorname{ind}_{\mu}(T)+\sum_{i=1}^{l} \frac{M}{k_{i} \mu_{i}} e_{i}\left[r\left(\underline{\lambda}^{i}\right)-r\left(\underline{\lambda}^{i-1}\right)\right](\bmod M),
$$

where $\underline{\lambda}^{i}=\underline{\lambda}^{i}(T)$ and $T$ ranges over the standard tableaux of shape $\underline{\lambda}$.

B. Generalized exponents. Following the notation of $\S 4$, write $G=$ $C_{m}$ and $G_{n}=C_{m} 2 S_{n}$. Since $C_{m}$ has been identified as the group of $m$ th roots of unity, we may thus identify $G_{n}$ as a subgroup of $\mathrm{GL}_{n}(\mathbf{C})$ consisting of pseudo permutation matrices. Via this representation, 
$G_{n}$ acts as a reflection group on $\mathbf{C}^{n}$. The corresponding ring of invariants in $S\left(\mathbf{C}^{n}\right)$ may be identified with the symmetric polynomials in the variables $x_{1}^{m}, \ldots, x_{n}^{m}$. In particular, the homogeneous degrees of $G_{n}$ are $m, 2 m, \ldots, n m$, as is well-known.

For any $\underline{\lambda}: I_{m} \rightarrow \mathscr{P}$ with $|\underline{\lambda}|=n$, let $G_{\underline{\lambda}}(q)$ denote the generating function for the generalized exponents of $G_{n}$ corresponding to $\underline{\lambda}$. If $x \in G_{n}$ is an $n$-cycle of $G$-class $\omega=e^{2 \pi i / m}$, it is easy to check that $x$ is $e^{2 \pi i / m n}$-regular. Hence, Theorem 2.2 implies $G_{\underline{\lambda}}(q)=P_{\underline{\lambda}, x}(q)$ $\left(\bmod 1-q^{m n}\right)$. In fact, we claim that $G_{\underline{\lambda}}(q)$ agrees with the description of $P_{\underline{\lambda}, x}(q)$ in Theorem 5.1 without reduction $\bmod 1-q^{m n}$; i.e.,

THEOREM 5.3. $G_{\underline{\lambda}}(q)=q^{r(\underline{\lambda})} I_{\underline{\lambda}}\left(q^{m}\right)$.

Proof. From (2.5) we have

$$
\frac{\left(q^{m}\right)_{n}}{\operatorname{det}(1-q x)}=\sum_{|\underline{\lambda}|=n} G_{\underline{\lambda}}(q) \chi^{\underline{\lambda}}(x)
$$

for all $x \in G_{n}$. Therefore, $\left(q^{m}\right)_{n}^{-1} G_{\underline{\lambda}}(q)$ is the (graded) multiplicity of $\chi^{\lambda}$ in the (graded) character $\psi_{n}: G_{n} \rightarrow \mathbf{C}[[q]]$ defined by

$$
\psi_{n}(x)=\operatorname{det}(1-q x)^{-1} \text {. }
$$

Consider the special case in which $\chi^{\lambda}$ is a pure wreath product of the form $\varphi^{k}<\chi^{\lambda}$ (i.e., the character of a $G_{n}$-module of the form $U<V$, where $\varphi^{k}=\operatorname{char} U$ and $\left.\chi^{\lambda}=\operatorname{char} V\right)$. Let $F_{k}^{\lambda}(q)$ denote the graded multiplicity of $\varphi^{k} 2 \chi^{\lambda}$ in $\psi_{n}$. We have

$$
F_{k}^{\lambda}(q)=\left\langle\varphi^{k}\left\langle\chi^{\lambda}, \psi_{n}\right\rangle=\frac{1}{n ! m^{n}} \sum_{x \in G_{n}} \frac{\varphi^{k} 2 \chi^{\lambda}(x)}{\operatorname{det}(1-q \bar{x})},\right.
$$

where $\bar{x}$ indicates complex conjugation of the matrix $x$.

LEMMA 5.4. $F_{k}^{\lambda}(q)=q^{k|\lambda|} s_{\lambda}\left(1, q^{m}, q^{2 m}, \ldots\right)=q^{k|\lambda|}\left(q^{m}\right)_{n}^{-1} I_{\lambda}\left(q^{m}\right)$.

Proof. For each $w \in S_{n}$, there are $m^{n}$ elements $x \in G_{n}$ whose underlying $S_{n}$-image is $w$. The $C_{m}$-classes corresponding to each cycle of $w$ are independently and uniformly distributed on $C_{m}$ among these $m^{n}$ choices. Therefore, if $w$ is of cycle-type $\mu$, Lemma 7.3 implies that the contribution of these elements to (5.3) is of the form

$$
\frac{1}{n !} \chi^{\lambda}(w) \prod_{i=1}^{\ell(\mu)} \frac{1}{m} \sum_{j=0}^{m-1} \frac{\varphi^{k}\left(\omega^{j}\right)}{1-\omega^{-j} q^{\mu_{i}}} .
$$


However,

$$
\frac{1}{m} \sum_{j=0}^{m-1} \frac{\varphi^{k}\left(\omega^{j}\right)}{1-\omega^{-j} q^{\mu_{i}}}=\sum_{r \geq 0}\left\langle\varphi^{k}, \varphi^{r}\right\rangle q^{r \mu_{i}}=q^{k \mu_{l}}\left(1-q^{m \mu_{i}}\right)^{-1},
$$

since the inner product $\left\langle\varphi^{k}, \varphi^{r}\right\rangle$ of $C_{m}$-characters vanishes unless $k=r$ $\bmod m$. Thus, (5.4) can be rewritten as

$$
\frac{1}{n !} \chi^{\lambda}(w) q^{k n} \operatorname{det}\left(1-q^{m} w\right)^{-1}
$$

if $w$ is represented as a permutation matrix. Therefore, (5.3) becomes

$$
F_{k}^{\lambda}(q)=\frac{1}{n !} q^{k n} \sum_{w \in S_{n}} \frac{\chi^{\lambda}(w)}{\operatorname{det}\left(1-q^{m} w\right)},
$$

which is an $S_{n}$-character inner product. By comparison with (3.2), it follows that

$$
F_{k}^{\lambda}(q)=q^{k n}\left(q^{m}\right)_{n}^{-1} G_{\lambda}\left(q^{m}\right),
$$

where $G_{\lambda}(q)$ is the usual generating function for generalized exponents of $S_{n}$. Apply Theorem 3.2.

To complete the proof of Theorem 5.3, recall that the general irreducible $G_{n}$-character is induced from one of the subgroups $G_{(n)}$ (Theorem 4.1). In particular, we note that

$$
\chi^{\underline{\lambda}}=\left(\varphi^{0} 2 \chi^{\lambda^{0}}\right) \times \cdots \times\left(\varphi^{m-1} 2 \chi^{\lambda^{m-1}}\right) \uparrow G_{n} .
$$

Since $\psi_{n} \downarrow G_{(n)}=\prod_{i=0}^{m-1} \psi_{n_{\imath}}$, an application of Frobenius reciprocity therefore yields

$$
\frac{1}{\left(q^{m}\right)_{n}} G_{\underline{\lambda}}(q)=\left\langle\chi^{\underline{\lambda}}, \psi_{n}\right\rangle_{G_{n}}=\prod_{i=0}^{m-1} F_{i}^{\lambda^{i}}(q)
$$

By Lemma 5.4, it follows that

$$
G_{\underline{\lambda}}(q)=q^{r(\underline{\lambda})}\left(q^{m}\right)_{n} \prod_{i=0}^{m-1} s_{\lambda^{i}}\left(1, q^{m}, q^{2 m}, \ldots\right) .
$$

Since $\prod_{i} s_{\lambda^{t}}$ is the skew Schur function indexed by the diagram of $\underline{\lambda}$, we may now deduce the desired result from Lemma 3.1.

In view of (3.3) and the preceding remark, we have the identity

$$
I_{\underline{\lambda}}(q)=(q)_{n} \prod_{i=0}^{m-1} \frac{q^{n\left(\lambda^{i}\right)}}{H_{\lambda^{i}}(q)}
$$

and hence, an explicit formula for the polynomials $G_{\underline{\lambda}}(q)$. 
For the hyperoctahedral group $B_{n}=C_{2} 2 S_{n}$ (i.e., $m=2$ ), the partition-valued functions $\underline{\lambda}$ may be identified with ordered pairs of partitions $(\lambda, \mu)$, and the polynomials $G_{(\lambda, \mu)}(q)$ are the fake degrees for the finite classical groups of type $B$. If we apply Theorem 5.3 and (5.6) to this case we obtain an explicit formula equivalent to Lusztig's [L, (2.4)].

COROLlary 5.5 .

$$
G_{(\lambda, \mu)}(q)=q^{|\mu|+2 n(\lambda)+2 n(\mu)} \frac{\left(q^{2}\right)_{n}}{H_{\lambda}\left(q^{2}\right) H_{\mu}\left(q^{2}\right)} .
$$

6. On reflection subgroups of $C_{m}\left\{S_{n}\right.$. Let $\delta: C_{m} \nmid S_{n} \rightarrow \mathbf{C}^{*}$ be the one-dimensional character defined so that for $x \in C_{m} \geqslant S_{n}, \delta(x)$ is the product of the nonzero entries of the $n \times n$ matrix $x$. (As usual, the nonzero entries are taken from the group of $m$ th roots of unity.) For any $d \mid m$, the kernel of $\delta^{d}$ forms a normal subgroup of index $m / d$ in $C_{m} \prec S_{n}$. Throughout this section, $m$ will be fixed. We will write $G_{n}$ for $C_{m} 2 S_{n}$ and $G_{n}(d)$ for $\operatorname{ker} \delta^{d}$. Note that in case $m=2, G_{n}(1)$ may be identified as the Weyl group $D_{n}$.

It is well-known that as a matrix group, $G_{n}(d)$ is generated by reflections acting (irreducibly, if $m>1$ ) on $\mathbf{C}^{n}$. In the classification of finite reflection groups due to Shephard and Todd [ST], one finds that, aside from the groups $G_{n}(d)$ and $S_{n}$, only a finite number of other irreducible reflection groups exist. Although $G_{n}(d)$ is not a wreath product, its irreducible representations still exhibit combinatorial structure, and so one may expect that its cyclic and generalized exponents should possess "natural" descriptions.

In Part B, we will derive explicit combinatorial interpretations for the generalized exponents of $G_{n}(d)$, as well as formulas for the associated generating functions.

To describe the cyclic exponents of $G_{n}(d)$ is more difficult in general. Via standard techniques of Clifford Theory, one may obtain the irreducible representations and characters of $G_{n}(d)$ from those of $G_{n}$. Through these techniques, one finds that the characters are described most naturally in an indirect fashion via discrete Fourier transforms. An outline that more fully explains this approach will be given in Part A. (In the special case corresponding to the Weyl groups $D_{n}$, these discrete Fourier transforms are the difference characters of $D_{n}$.)

The indirectness of this approach to the characters of $G_{n}(d)$ forces us to describe the cyclic exponents indirectly as well, and thus deviate 
from our original program. We therefore do not attempt to carry out the techniques of Part A in their full generality. Instead, we restrict our attention to the Weyl groups $D_{n}$ (Part C). The cyclic exponents of these groups have a level of complexity sufficient to convey the flavor of the general case, but they are still simple enough to be comprehensible.

A. Clifford theory. Let $H$ be a normal subgroup of a finite group $G$ such that $G / H$ is cyclic. Of course, the example we have in mind is the case $G=G_{n}$ and $H=G_{n}(d)$, but it will be convenient to begin in this more general setting. Let $C \cong G / H$ denote the (cyclic) group of one-dimensional $G$-characters $\delta$ with $\operatorname{ker} \delta \supseteq H$. Note that $C$ acts on (the isomorphism classes of) irreducible $G$-modules via $V \stackrel{\delta}{\longmapsto} \delta \otimes V$. Two irreducible $G$-modules are said to be associates if they appear in the same $C$-orbit.

Choose a fixed irreducible $G$-module $V$, and let

$$
C_{V}=\{\delta \in C: V \cong \delta \otimes V\}
$$

denote the stabilizer of $V$. Let $\delta \in C$ be a generator of $C_{V}$. There must exist linear transformations $S \in \mathrm{GL}(V)$ which explicitly demonstrate the isomorphism $V \cong \delta \otimes V$; i.e.,

$$
S g v=\delta(g) g S v
$$

for all $g \in G, v \in V$. If $\left|C_{V}\right|=k$, then $\delta$ is of order $k, S^{k}$ commutes with the action of $G$, and so Schur's Lemma implies that $S^{k}$ is a scalar. We will always assume $S$ is normalized so that $S^{k}=1$, and we call $S$ an associator for $V$. A further application of Schur's Lemma easily shows that $S$ is unique, aside from the fact that $\omega S$, for any $k$ th root of unity $\omega$, is also an associator for $V$.

Choose a fixed associator $S$ for $V$, and let

$$
V=E_{0} \oplus E_{1} \oplus \cdots \oplus E_{k-1}
$$

denote the eigenspace decomposition of $S$ on $V$, where

$$
E_{j}=\left\{v \in V: S v=\omega^{j} v\right\}
$$

and $\omega=e^{2 \pi i / k}$. Since $\operatorname{ker} \delta \supseteq H,(6.1)$ implies that each eigenspace is an $H$-module.

The following result summarizes the relationship between irreducible $G$-modules and $H$-modules. Since $G / H$ is assumed to be cyclic, we may draw stronger conclusions than those that normally occur in Clifford's Theorem (e.g. [CR, (11.1)]). Although these stronger conclusions are presumably a well-known part of Clifford Theory, we have included a proof since it is difficult to find in the literature. 
Proposition 6.1. Let $G, H$ and $V$ be as above.

(a) $G$ acts transitively on the eigenspaces $E_{j}$ in (6.2).

(b) The $E_{j}$ 's are nonzero, inequivalent, irreducible $H$-modules.

(c) $E_{j} \uparrow G$ is the direct sum of the (distinct) associates of $V$.

Proof. (a) This follows directly from (6.1).

(b) Since $G$ acts transitively on the $E_{j}$ 's, they must all be nonzero. In particular, the $H$-module structure of $V$ has at least $k$ irreducible constituents. To prove that the $E_{j}$ 's are irreducible and inequivalent, it therefore suffices to show $\|\chi\|_{H}^{2}=k$, where $\chi=\operatorname{char} V$ and $\|\cdot\|_{H}$ denotes the $H$-character metric.

Since $k=\left|C_{V}\right|$, it follows that $V$ has $|C| / k$ distinct associates, and each of these occur with multiplicity $k$ among $\{\varepsilon \otimes V: \varepsilon \in C\}$. Therefore,

$$
\left\|\sum_{\varepsilon \in C} \varepsilon \chi\right\|_{G}^{2}=k^{2} \cdot \frac{|C|}{k}=k \cdot|C| .
$$

However, by the orthogonality of cyclic group characters,

$$
\sum_{\varepsilon \in C} \varepsilon(g)= \begin{cases}|C| & \text { if } g \in H \\ 0 & \text { if } g \notin H,\end{cases}
$$

so we have

$$
\left\|\sum_{\varepsilon \in C} \varepsilon \chi\right\|_{G}^{2}=\frac{1}{|G|} \sum_{h \in H}|C|^{2} \cdot|\chi(h)|^{2}=|C| \cdot\|\chi\|_{H}^{2} .
$$

Upon comparison with (6.3), we find $\|\chi\|_{H}^{2}=k$.

(c) Frobenius reciprocity implies that $E_{j} \uparrow G$ is a direct sum (possibly with multiplicity) of irreducible $G$-modules that include $E_{j}$ as an $H$ submodule. We know that this list includes the $|C| / k$ associates of $V$, since they are all identical as $H$-modules. However,

$$
\frac{|C|}{k} \cdot \operatorname{dim} V=|C| \cdot \operatorname{dim} E_{j}=\operatorname{dim}\left(E_{j} \uparrow G\right),
$$

so this list spans all of $E_{j} \uparrow G$.

REMARK. As a corollary to this result, we see that there is a one-toone correspondence between the irreducible $H$-characters and ordered pairs $(\mathscr{O}, \varepsilon)$ consisting of a $C$-orbit $\mathscr{O}$ of irreducible $G$-characters and a character $\varepsilon \in C$ that stabilizes $\mathscr{O}$. 
Let $\chi_{j}$ denote the character of $H$ on $E_{j}$. The discrete Fourier transform (DFT) of $\chi=$ char $V$ with respect to $S$ is the family of $H$-class functions $\Delta_{i}(h): H \rightarrow \mathrm{C}^{*}$ defined by

$$
\Delta_{i}(h)=\operatorname{tr}\left(\left.S^{i} h\right|_{V}\right)=\sum_{j=0}^{k-1} \omega^{i j} \chi_{j}(h) .
$$

Note that the $\Delta_{i}$ 's contain sufficient information to recover the $\chi_{j}$ 's. Specifically, we have

$$
\chi_{j}(h)=\frac{1}{k} \sum_{i=0}^{k-1} \omega^{-i j} \Delta_{i}(h),
$$

by the orthogonality of cyclic group characters. In case the stabilizer $C_{V}$ is of order 2 (i.e., $k=2$ ), we have $\Delta_{0}=\chi_{0}+\chi_{1}$ and $\Delta_{1}$ is the difference character: $\Delta_{1}=\chi_{0}-\chi_{1}$.

The following result shows that to describe the DFT of a $G$-character $\chi$, it is sufficient to evaluate $\Delta_{i}(h)$ for one element from each conjugacy class of $G$ in $H(0 \leq i<k)$.

Proposition 6.2. If $g \in G, h \in H$, then $\Delta_{i}\left(g h g^{-1}\right)=\delta^{i}(g) \Delta_{i}(h)$.

Proof. Let $\delta(g)=\omega^{r}$. Note that (6.1) implies $g E_{j}=E_{j+r}$, and hence

$$
\chi_{j+r}\left(g h g^{-1}\right)=\chi_{j}(h)
$$

Therefore,

$$
\begin{aligned}
\Delta_{i}\left(g h g^{-1}\right) & =\delta^{i}(g) \sum_{j=0}^{k-1} \omega^{i(j-r)} \chi_{j}\left(g h g^{-1}\right) \\
& =\delta^{i}(g) \sum_{j=0}^{k-1} \omega^{i j} \chi_{j+r}\left(g h g^{-1}\right)=\delta^{i}(g) \Delta_{i}(h) .
\end{aligned}
$$

In summary, to determine the irreducible characters of $H$ from those of $G$, it suffices to choose one irreducible $G$-module $V$ from each $C$-orbit, construct an associator $S$ for $V$, and evaluate the DFT $\Delta_{i}(h)=$ $\operatorname{tr}\left(\left.S^{i} h\right|_{V}\right)$ for one element from each $G$-class in $H$. This technique can be carried out explicitly for the pair $G=G_{n}, H=G_{n}(d)$; in $\S 7 \mathrm{~B}$ we treat the case $G=B_{n}, H=D_{n}$ in detail.

B. Generalized exponents. As in the introduction of $\S 6$, let $\delta(x)$ denote the product of the nonzero entries of $x \in G_{n} \subset \mathrm{GL}_{n}(\mathbf{C})$. Note 
that the character group $C$ with respect to the normal subgroup $G_{n}(d)$ (cf. Part A) is in this case cyclic of order $m / d$ and generated by $\delta^{d}$. Recall $(\S 5)$ that the irreducible $G_{n}$-characters are indexed by partitionvalued functions $\underline{\lambda}: I_{m} \rightarrow \mathscr{P}$ with $|\underline{\underline{ }}|=n$. The action of $C$ on irreducible $G$-modules therefore yields an action of $C$ on the corresponding indices $\underline{\lambda}$. If we regard $\underline{\lambda}$ as an $m$-tuple $\left(\ldots, \lambda^{1}, \lambda^{0}\right)$ of partitions, then the $C$-orbit $\mathscr{O}$ of $\underline{\lambda}$ consists of the $d$-fold cyclic shifts of $\underline{\lambda}$. (We choose to list the partitions $\lambda^{i}$ in reverse order for reasons that will become apparent later). The orbit $\mathscr{O}$ may be visualized as a necklace of $m / d$ beads consisting of $d$-tuples of partitions.

Let $\chi$ be an irreducible $G_{n}(d)$-character, and let $G_{\chi}(q)$ denote the usual generating function for the generalized $\chi$-exponents. According to Proposition 6.1(c), there must be a $C$-orbit $\mathscr{O}$ of irreducible $G_{n^{-}}$ characters such that

$$
\chi \uparrow G_{n}=\sum_{\underline{\lambda} \in \mathcal{O}} \chi^{\underline{\lambda}}
$$

The following result shows that $G_{\chi}(q)$ may be expressed in terms of the generalized exponent polynomials $G_{\underline{\lambda}}(q)$ corresponding to the $G_{n}$ characters indexed by $\underline{\lambda} \in \mathscr{O}$.

Proposition 6.3. If $\chi$ is a $G_{n}(d)$-character associated with the orbit $\mathscr{O}$, then

$$
G_{\chi}(q)=\frac{1-q^{d n}}{1-q^{m n}} \sum_{\underline{\lambda} \in \mathscr{O}} G_{\underline{\lambda}}(q) .
$$

Proof. The reflection representation of $G_{n}(d)$ on $\mathbf{C}^{n}$ is clearly a restriction of the corresponding representation of $G_{n}$. The same is true for the associated symmetric algebra $S\left(\mathbf{C}^{n}\right)$. If we identify $S\left(\mathbf{C}^{n}\right)$ with $\mathbf{C}\left[x_{1}, \ldots, x_{n}\right]$, then the $G_{n}(d)$-invariants may be identified as the subalgebra generated by $\left(x_{1} \cdots x_{n}\right)^{d}$ and the elementary symmetric polynomials in the variables $x_{1}^{m}, \ldots, x_{n}^{m}$. The homogeneous degrees of $G_{n}(d)$ are therefore $m, 2 m, \ldots,(n-1) m, n d$ [ST]. Hence, we may rewrite $(2.5)$ in the form

$$
\frac{1}{\left(q^{m}\right)_{n}} \frac{1-q^{m n}}{1-q^{d n}} G_{\chi}(q)=\left\langle\chi, \psi_{n} \downarrow G_{n}(d)\right\rangle,
$$

where $\psi_{n}$ is the graded $G_{n}$-character of $S\left(\mathbf{C}^{n}\right)$, as in (5.2). By Frobenius reciprocity, (6.5) therefore implies

$$
\frac{1}{\left(q^{m}\right)_{n}} \frac{1-q^{m n}}{1-q^{d n}} G_{\chi}(q)=\sum_{\underline{\lambda} \in \mathscr{O}}\left\langle\chi^{\underline{\lambda}}, \psi_{n}\right\rangle=\frac{1}{\left(q^{m}\right)_{n}} \sum_{\underline{\lambda} \in \mathscr{O}} G_{\underline{\lambda}}(q) .
$$


For each $C$-orbit $\mathscr{O}$, let $R_{\mathscr{O}}(q)$ denote the polynomial

$$
R_{\mathscr{O}}(q)=\sum_{\underline{\lambda} \in \mathscr{O}} q^{r(\underline{\lambda})}
$$

where $r(\underline{\lambda})$ is the statistic (5.1). Since Proposition 6.3 shows that $G_{\chi}$ depends only on $\mathscr{O}$, we may write $G_{\mathscr{O}}(q)$ as an unambiguous abbreviation for $G_{\chi}(q)$. Let $\{\underline{\lambda}\}$ denote the $C$-orbit of $\underline{\lambda}$. From Theorem 5.3 we may deduce

COROllary 6.4 .

$$
G_{\{\underline{\lambda}\}}(q)=\frac{1-q^{d n}}{1-q^{m n}} R_{\{\underline{\lambda}\}}(q) I_{\underline{\lambda}}\left(q^{m}\right) .
$$

Since there is an explicit formula for $I_{\underline{\lambda}}(q)$ (viz., (5.6)), we now have an explicit formula for $G_{\{\underline{\lambda}\}}(q)$. For the Weyl groups $D_{n}$ (i.e., $m=2, d=1)$, the $C$-orbits consist of unordered pairs of partitions $\{\lambda, \mu\}$ with $|\lambda|+|\mu|=n$. The stabilizer of such an orbit is nontrivial iff $\lambda=\mu$. If we specialize (5.6) and Corollary 6.4 to $D_{n}$, we obtain a formula for the fake degrees for finite classical groups of type $D$ originally due to Lusztig $[\mathbf{L},(2.4,5)]$.

COROLlaRy 6.5. If $|\lambda|=k,|\mu|=n-k$, then

$$
G_{\{\lambda, \mu\}}(q)=\kappa_{\lambda \mu} q^{2 n(\lambda)+2 n(\mu)} \frac{q^{k}+q^{n-k}}{1+q^{n}} \frac{\left(q^{2}\right)_{n}}{H_{\lambda}\left(q^{2}\right) H_{\mu}\left(q^{2}\right)},
$$

where $\kappa_{\lambda \mu}=1 / 2$ if $\lambda=\mu ; \kappa_{\lambda \mu}=1$ if $\lambda \neq \mu$.

For any tableau $T$ of shape $\underline{\lambda}$, let $T_{j}$ denote the restriction of $T$ to the subdiagram of shape $\lambda^{j}(0 \leq j<m)$, and identify $T$ with the $m$-tuple $\left(\ldots, T_{1}, T_{0}\right)$. The group $C$ clearly acts on the set of $m$-tuples of tableaux (of arbitrary shape) via $d$-fold cyclic shifts; we will use the notation $\{T\}$ for the $C$-orbit of $T$, and we will refer to $\{T\}$ as a tableau of shape $\{\underline{\lambda}\}$.

In the following analysis, we will need to consider $m$-tuples $T=$ $\left(\ldots, T_{1}, T_{0}\right)$ of tableaux, possibly with repeated entries, in which any given letter may appear in at most one component tableau. In that case, there is a unique component $T_{k}$ that contains the largest entry of $T$. If $k$ is minimal (i.e., the largest entry occurs as far as possible to the right) among all candidates in the $C$-orbit $\{T\}$, then $T$ is said to be the canonical representative of $\{T\}$. For example, the tableau 
in Figure $4(\mathrm{c})$ is a canonical representative if $d=2$ or 3 , but not if $d=1$.

Let $T$ be a standard tableau of shape $\underline{\lambda}$, and assume $T$ is the canonical representative of the orbit $\{T\}$. We write $r\{T\}$ as an abbreviation for $r(\underline{\lambda})$. If $j$ appears in a column to the right of $j+1$, or in the same column as $j+1$ in the $m$-tuple $T=\left(\ldots, T_{1}, T_{0}\right)$, then $j$ is said to be a descent in $\{T\}$. We use the notation $D\{T\}$ for the set of descents in $T$, and the index of the orbit $\{T\}$ is defined via

$$
\operatorname{ind}\{T\}=\sum_{j \in D\{T\}} j .
$$

We remark that if $T$ is embedded in $\mathbf{Z}^{2}$ so that the columns of $T$ appear in the same order as the columns in $\left(\ldots, T_{1}, T_{0}\right)$, then these descent sets and indices coincide with those of $\S 3 \mathrm{~A}$. In the example in Figure 4(c), we have $D\{T\}=\{1,4,5,7,10\}$, ind $\{T\}=27$, and $r\{T\}=28$, assuming $d=2$ or 3 .

THEOREM 6.6. The generalized exponents of $G_{n}(d)$ corresponding to $\{\underline{\lambda}\}$ are

$$
r\{T\}+m \cdot \operatorname{ind}\{T\},
$$

where $\{T\}$ ranges over the standard tableaux of shape $\{\underline{\lambda}\}$.

Proof. Let $\underline{\lambda}$ be an $m$-tuple of partitions with $|\underline{\lambda}|=n$. It will be convenient to regard the diagram $D_{\underline{\lambda}}$ as a subset of $\mathbf{Z}^{3}$; i.e.,

$$
D_{\underline{\lambda}}=\left\{(i, j, k):(i, j) \in D_{\lambda^{k}}, 0 \leq k<m\right\} .
$$

We will use this version of $D_{\underline{\lambda}}$ as the domain for tableaux of shape $\underline{\lambda}$.

In view of (5.5), we have

$$
\frac{1}{\left(q^{m}\right)_{n}} G_{\underline{\lambda}}(q)=\prod_{k=0}^{m-1} s_{\lambda^{k}}\left(q^{k}, q^{m+k}, q^{2 m+k}, \ldots\right) .
$$

Therefore, $\left(q^{m}\right)_{n}^{-1} G_{\underline{\lambda}}(q)$ may be interpreted as the generating function for tableaux $T$ of shape $\underline{\lambda}$ satisfying $T(i, j, k)=k \bmod m$ and $T(i, j, k) \geq 0$. As in the proof of Lemma 3.1, it will be necessary to modify this interpretation slightly by using reverse tableaux. If we apply this interpretation to Proposition 6.3, we discover that if $\mathscr{O}$ is any $C$-orbit of partitions, then

$$
\frac{1}{\left(q^{m}\right)_{n-1}\left(1-q^{d n}\right)} G_{\mathscr{O}}(q)=\sum_{\{T\}} q^{|T|},
$$


summed over all reverse tableaux $\{T\}$ of shape $\mathscr{O}$ satisfying

(1) $T(i, j, k)-T\left(i^{\prime}, j^{\prime}, k^{\prime}\right)=k-k^{\prime}(\bmod m)$,

(2) $T(i, j, k) \geq 0$ and $T(i, j, k)=k(\bmod d)$,

where $|T|$ denotes the sum of the entries of $T$. An example with $m=$ $6, d=2$, and shape $\underline{\lambda}=(1,21,11,1,21,11)$ appears in Figure 4(a).

Note that properties (1) and (2) are invariant under the action of $C$, so they may be imposed on any member of the orbit $\{T\}$ without conflict. Henceforth, we will always assume that $T$ is chosen to be the canonical representative of $\{T\}$. (Note that since we are using reverse tableaux, the position of the numerically smallest entry of $T$ determines whether $T$ is canonical.) Given such a tableau $T$, define a new tableau $\hat{T}$ of the same shape by setting $\hat{T}(i, j, k)=T(i, j, k)-k$, and observe that the entries of $\hat{T}$ are characterized by

(i) $\hat{T}(i, j, k)=\hat{T}\left(i^{\prime}, j^{\prime}, k^{\prime}\right)(\bmod m)$,

(2) $\hat{T}(i, j, k) \geq 0$ and $\hat{T}(i, j, k)=0(\bmod d)$.

The weight of $\hat{T}$ is clearly $|T|-r\{T\}$.

Assuming $T$ is of shape $\underline{\lambda}$, define a total order on the cells of $D_{\underline{\lambda}}$ as follows:

$$
\begin{aligned}
& (i, j, k)<\left(i^{\prime}, j^{\prime}, k^{\prime}\right) \\
& \quad \text { iff }\left\{\begin{array}{l}
\hat{T}(i, j, k)>\hat{T}\left(i^{\prime}, j^{\prime}, k^{\prime}\right) \text {, or } \\
\hat{T}(i, j, k)=\hat{T}\left(i^{\prime}, j^{\prime}, k^{\prime}\right) \text { and }(j, k)<_{L}\left(j^{\prime}, k^{\prime}\right),
\end{array}\right.
\end{aligned}
$$

where $<_{L}$ denotes the following lexicographic order:

$$
(j, k)<_{L}\left(j^{\prime}, k^{\prime}\right) \text { iff }\left\{\begin{array}{l}
k>k^{\prime}, \text { or } \\
k=k^{\prime} \text { and } j<j^{\prime} .
\end{array}\right.
$$

Use this total order to number the cells of $D_{\lambda}$ from 1 to $n$, and thus produce a standard tableau $S$ of shape $\underline{\lambda}$. In Figures $4(a)-4(\mathrm{c})$ is an illustration of the operations $T \mapsto \hat{T}$ and $\hat{T} \mapsto S$. Note that $S$ is the canonical representative of $\{S\}$.

Let $\alpha_{i}$ denote the entry of $\hat{T}$ in the cell numbered $i$ by $S$. Given $S$ and $\alpha$, one may easily recover $\hat{T}$ (and hence, $T$ ), so the map $T \mapsto$ $\hat{T} \mapsto(S, \alpha)$ is injective. For a fixed choice of $S$, the possible sequences $\alpha$ that arise in this fashion are characterized by (cf. the proof of Lemma 3.1):

(1) $\alpha_{1} \geq \cdots \geq \alpha_{n} \geq 0$

(2) $\alpha_{k}>\alpha_{k+1}$ if $k \in D\{S\}$,

(3) $\alpha_{1}=\cdots=\alpha_{n}(\bmod m), \alpha_{n}=0(\bmod d)$.

Therefore, if we subtract $m$ from $\alpha_{1}, \ldots, \alpha_{k}$ for each $k \in D\{S\}$, we obtain a partition $\alpha^{*}$ whose terms are characterized by (1) and (3), 


\begin{tabular}{|c|c|}
\hline 55 & $\begin{array}{ll}66 & 18 \\
6 & \end{array}$ \\
\hline
\end{tabular}

FIGURE 4(a)

$\begin{array}{llllllll}50 & 62 & 14 & 32 & 14 & 38 & 26 & 38 \\ & 2 & 26 & & 2 & 8\end{array}$

FIGURE 4(b)

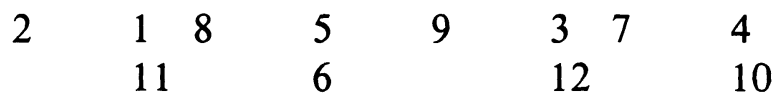

FIGURE 4(c)

and whose weight satisfies

$$
\left|\alpha^{*}\right|=|\alpha|-m \cdot \operatorname{ind}\{S\} .
$$

Since the generating function for $\alpha^{*}$ is $\left(q^{m}\right)_{n-1}^{-1}\left(1-q^{d n}\right)^{-1}$, it follows that for fixed $S$,

$$
\sum_{T \mapsto(S, \cdot)} q^{|T|}=\sum_{\alpha^{*}} q^{\left|\alpha^{*}\right|+m \cdot \operatorname{ind}\{S\}+r\{S\}}=\frac{q^{m \cdot \operatorname{ind}\{S\}+r\{S\}}}{\left(q^{m}\right)_{n-1}\left(1-q^{d n}\right)} .
$$

Apply (6.6) to complete the proof.

C. The cyclic exponents of $D_{n}$. According to Proposition 6.1, an irreducible character $\chi^{(\lambda, \mu)}$ of the hyperoctahedral group $B_{n} \cong C_{2} \nmid S_{n}$ is either an irreducible $D_{n}$-character (the case $\lambda \neq \mu$ ), or the sum of two such characters (the case $\lambda=\mu$ ). In the latter case, we will use the notation $\{\lambda, \lambda\}_{ \pm}$to index the two irreducible constituents, so that

$$
\chi^{(\lambda, \lambda)} \downarrow D_{n}=\chi_{+}^{\{\lambda, \lambda\}}+\chi_{-}^{\{\lambda, \lambda\}} \text {. }
$$

This indexing scheme is slightly ill-defined at present, since we have made no attempt to distinguish between the two constituents. An explicit choice will be made in $\S 7 \mathrm{~B}$. Given such a choice, let

$$
\Delta^{\lambda}=\chi_{+}^{\{\lambda, \lambda\}}-\chi_{-}^{\{\lambda, \lambda\}}
$$

denote the associated difference character. In Theorem 7.5, we show that $\Delta^{\lambda}$ is closely related to the symmetric group character $\chi^{\lambda}$.

Since $D_{n}$ is a subgroup of $B_{n}$, it follows that the cyclic exponents of $D_{n}$ with respect to $\chi^{(\lambda, \mu)}$ may be obtained as mere special cases of the results in $5 \mathrm{~A}$ (with $m=2$ ). Therefore, to assemble a complete 
description of the cyclic exponents of $D_{n}$, we need only to determine how to distribute the (known) eigenvalues of the $B_{n}$-module indexed by $(\lambda, \lambda)$ between the two $D_{n}$-modules indexed by $\{\lambda, \lambda\}_{ \pm}$. In short, it suffices to determine the polynomials

$$
Q_{\lambda, x}(q):=P_{\{\lambda, \lambda\}_{+}, x}(q)-P_{\{\lambda, \lambda\}_{-}, x}(q),
$$

for each $x \in D_{n}$.

Henceforth, it will be more convenient to assume that $|\lambda|=n$, so that the indices $\{\lambda, \lambda\}_{ \pm}$label representations of $D_{2 n}$. Likewise, we insist that $x \in D_{2 n}$.

To describe the $Q_{\lambda, x}$ 's, define a sequence of polynomials $c_{n}(q)$ via

$$
c_{n}(q)=(1-q)\left(1-q^{3}\right) \cdots\left(1-q^{2 n-1}\right),
$$

and extend the notation to partitions $\mu$ by defining

$$
c_{\mu}(q)=c_{\mu_{1}}\left(q^{m / \mu_{1}}\right) c_{\mu_{2}}\left(q^{m / \mu_{2}}\right) \cdots,
$$

where $m=\left[\mu_{1}, \mu_{2}, \ldots\right]$ (least common multiple). Recall ( $\left.\S 3\right)$ that $P_{\lambda, \mu}(q)$ denotes the cyclic exponent polynomial for any $w \in S_{n}$ of cycle-type $\mu$ with respect to the $S_{n}$-module indexed by $\lambda$.

THEOREM 6.7. Let $|\lambda|=n, x \in D_{2 n}$.

(a) $Q_{\lambda, x}(q)=0$ unless every cycle of $x$ is of even length and $C_{2}$-class +1 .

(b) If $x$ is a permutation matrix of cycle-type $2 \mu$, then

$$
Q_{\lambda, x}(q)=c_{\mu}(q) P_{\lambda, \mu}\left(q^{2}\right) \quad\left(\bmod 1-q^{2 m}\right),
$$

where $2 m=\left[2 \mu_{1}, 2 \mu_{2}, \ldots\right]$ denotes the order of $x$.

Proof. (a) Note that Proposition 6.2 implies

$$
\Delta^{\lambda}\left(y x y^{-1}\right)=\delta(y) \Delta^{\lambda}(x)
$$

for $y \in B_{2 n}, x \in D_{2 n}$. If the centralizer $C_{B_{2 n}}(x)$ includes an element $y$ with $\delta(y)=-1$, it follows that $\Delta^{\lambda}=0$ on $\langle x\rangle$, and hence, $Q_{\lambda, x}(q)=0$. Since $C_{B_{2 n}}(x)$ includes the cycles of $x$, the existence of such $y$ is clear if any cycle of $x$ is of $C_{2}$-class -1 . If some cycle $x_{0}$ is of odd length, take $y \in C_{B_{2 n}}(x)$ to be the diagonal matrix which acts as -1 on the domain of $x_{0}$ and as 1 on the elements outside the domain of $x_{0}$. Thus (a) follows.

(b) Let $x \in D_{2 n}$ be a permutation matrix of order $2 m$ and cycle-type $2 \mu$. Set $\omega=e^{\pi i / m}$. 
LEMMA 6.8. If $x^{j}$ is a product of $l$ disjoint cycles, then $c_{\mu}\left(\omega^{j}\right)=2^{l}$ if every cycle of $x^{j}$ is of even length; otherwise, $c_{\mu}\left(\omega^{j}\right)=0$.

Proof. From the multiplicative definition of $c_{\mu}$, it is easy to reduce to the case in which $x$ is a $2 n$-cycle. Under this assumption, there are $l=(2 n, j)$ cycles in $x^{j}$, and they are all of the same length, namely, $k=2 n /(2 n, j)$. If $k$ is odd, then $\omega^{j}$ is a $2 n$th root of unity of odd order, and the definition (6.7) shows $c_{\mu}\left(\omega^{j}\right)=0$ in that case. If $k$ is even, say $k=2 r$, we have

$$
c_{n}(q)=\frac{(q)_{2 n}}{\left(q^{2}\right)_{n}}=\left[\frac{(q)_{2 r}}{\left(q^{2}\right)_{r}}\right]^{l}=\left[\frac{(q)_{2 r-1}}{\left(q^{2}\right)_{r-1}}\right]^{l} \quad\left(\bmod 1-q^{2 r}\right) .
$$

Since $\omega^{j}$ is a primitive $2 r$ th root of unity, we have $(q)_{2 r-1}=2 r$ and $\left(q^{2}\right)_{r-1}=r$ at $q=\omega^{j}$, and therefore, $c_{\mu}\left(\omega^{j}\right)=2^{l}$.

To complete the proof of $(b)$, note that Proposition 1.1 shows that it suffices to prove

$$
\Delta^{\lambda}\left(x^{j}\right)=c_{\mu}\left(\omega^{j}\right) P_{\lambda, \mu}\left(\omega^{2 j}\right)
$$

for $0 \leq j<2 m$. In case any cycle of $x^{j}$ is of odd length, the proof of (a) shows that $\Delta^{\lambda}\left(x^{j}\right)=0$, and Lemma 6.8 shows that $c_{\mu}\left(\omega^{j}\right)=0$, so (6.8) is verified. In the remaining case, we may assume that $x^{j}$ is of cycle-type $2 \nu$ for some partition $\nu$. In that case, any $w \in S_{n}$ of cycle-type $\mu$ has the property that $w^{j}$ is of cycle-type $\nu$. Hence, $P_{\lambda, \mu}\left(\omega^{2 j}\right)=\chi^{\lambda}(\nu)$, and to prove (6.8) reduces to verifying

$$
\Delta^{\lambda}\left(x^{j}\right)=2^{\ell(\nu)} \chi^{\lambda}(\nu),
$$

which is a consequence of Theorem 7.5.

\section{Appendix.}

A. A Murnaghan-Nakayama rule for $G$ ? $S_{n}$. In this section, we prove the recurrence for evaluating characters of $G_{n}=G \nmid S_{n}$ stated in Theorem 4.3. As a first step, we generalize the branching rule (3.4).

Lemma 7.1. Let $\underline{\lambda}: I_{G} \rightarrow \mathscr{P}$ and $|\underline{\lambda}|=n$. We have

$$
\chi^{\underline{\lambda}} \downarrow G_{r} \times G_{n-r}=\sum_{|\underline{\mu}|=r} \chi^{\underline{\underline{\mu}}} \times \chi^{\underline{\lambda}}-\underline{\underline{\mu}},
$$

summed over $\underline{\mu}: I_{G} \rightarrow \mathscr{P}$ with $\underline{\mu} \subseteq \underline{\lambda}$.

Proof. Let $|\underline{\mu}|=r,|\underline{\nu}|=n-r$, and let $c(\underline{\lambda}, \underline{\mu}, \underline{\nu})$ denote the multiplicity of $\chi^{\underline{\underline{\nu}}}$ in $\chi^{\lambda /}-\underline{\mu}$. From the definition of $\chi^{\underline{\lambda}}-\underline{\underline{\mu}}$, we have

$$
c(\underline{\lambda}, \underline{\mu}, \underline{\nu})=\prod_{\theta \in I_{G}} c\left(\lambda^{\theta}, \mu^{\theta}, \nu^{\theta}\right)
$$


where $c\left(\lambda^{\theta}, \mu^{\theta}, \nu^{\theta}\right)$ denotes the multiplicity of (the symmetric group character) $\chi^{\nu^{\theta}}$ in $\chi^{\lambda^{\theta} / \mu^{\theta}}$, as defined in (3.5). We claim that $c(\underline{\lambda}, \underline{\mu}, \underline{\nu})$ is also the multiplicity of $\chi^{\lambda}$ in the character $\left(\chi^{\mu}-\times \chi^{\nu}\right) \uparrow G_{n}$ induced from $G_{r} \times G_{n-r}$. If so, then an application of Frobenius reciprocity would yield

and thus prove the lemma.

$$
\begin{aligned}
\chi^{\underline{\lambda}} \downarrow G_{r} \times G_{n-r} & =\sum_{|\underline{\mu}|=r} \sum_{|\underline{\nu}|=n-r} c(\underline{\lambda}, \underline{\mu}, \underline{\nu}) \chi^{\underline{\mu}} \times \chi^{\underline{\nu}} \\
& =\sum_{|\underline{\mu}|=r} \chi^{\underline{\mu}} \times \chi^{\lambda-} \underline{\mu},
\end{aligned}
$$

To prove the claim, we may suppose that

$$
\begin{aligned}
& \chi^{\underline{\mu}}=\operatorname{char}\left[\left(U_{1} \prec V_{1}\right) \otimes \cdots \otimes\left(U_{t} \prec V_{t}\right) \uparrow G_{r}\right], \\
& \chi^{\underline{\nu}}=\operatorname{char}\left[\left(U_{1} \prec W_{1}\right) \otimes \cdots \otimes\left(U_{t} \prec W_{t}\right) \uparrow G_{n-r}\right]
\end{aligned}
$$

for suitable symmetric group modules $V_{i}, W_{i}$ (cf. (4.1)). Since outer tensor products commute with induction [CR, (10.7)], we have

$$
\chi^{\underline{\mu}} \times \chi^{\underline{\nu}}=\operatorname{char}\left[\left(U_{1} \curlywedge\left(V_{1} \otimes W_{1}\right)\right) \otimes \cdots \otimes\left(U_{t} \succ\left(V_{t} \otimes W_{t}\right)\right) \uparrow G_{r} \times G_{n-r}\right] .
$$

Using the transitivity of induction [CR, (10.6)] and the fact that

$$
U<\left[(V \otimes W) \uparrow S_{n}\right] \cong[U>(V \otimes W)] \uparrow G_{n}
$$

for $S_{r}$-modules $V$ and $S_{n-r}$-modules $W$ [M2, (6.8)], it follows that $\left(\chi^{\underline{\mu}} \times \chi^{\nu}\right) \uparrow G_{n}=\operatorname{char}\left[\left(U_{1} \gamma\left(V_{1} \otimes W_{1} \uparrow S_{n_{1}}\right)\right) \otimes \cdots \otimes\left(U_{t} 2\left(V_{t} \otimes W_{t} \uparrow S_{n_{t}}\right)\right) \uparrow G_{n}\right]$, where $n_{i}=\left|\mu^{\theta}\right|+\left|\nu^{\theta}\right|$ if $\theta=$ char $U_{i}$. Therefore, the multiplicity of $\chi^{\lambda}$ in $\left(\chi^{\underline{\mu}} \times \chi^{\nu}\right) \uparrow G_{n}$ is

$$
\prod_{\theta \in I_{G}}\left\langle\chi^{\lambda^{\theta}},\left(\chi^{\mu^{\theta}} \times \chi^{\nu^{\theta}}\right) \uparrow S_{n_{\theta}}\right\rangle
$$

where $\langle\cdot, \cdot\rangle$ denotes the inner product of symmetric group characters. This multiplicity is indeed $c(\underline{\lambda}, \underline{\mu}, \underline{\nu})$, since (3.4) and Frobenius reciprocity imply

$$
\left\langle\chi^{\lambda^{\theta}},\left(\chi^{\mu^{\theta}} \times \chi^{\nu^{\theta}}\right) \uparrow S_{n_{\theta}}\right\rangle=c\left(\lambda^{\theta}, \mu^{\theta}, \nu^{\theta}\right) .
$$
have

COROLlaRY 7.2. Let $\underline{\lambda}, \underline{\nu}: I_{G} \rightarrow \mathscr{P},|\underline{\lambda}|=n, \underline{|\nu|}=s$, and $\underline{\nu} \subseteq \underline{\lambda}$. We

$$
\chi^{\lambda-\underline{\nu}}-\downarrow G_{r-s} \times G_{n-r}=\sum_{|\underline{\mu}|=r} \chi^{\underline{\mu}}-\underline{\nu} \times \chi^{\lambda / \mu}-\underline{\mu},
$$

summed over $\underline{\mu}: I_{G} \rightarrow \mathscr{P}$ with $\underline{\nu} \subseteq \underline{\mu} \subseteq \underline{\lambda}$. 
Proof. By Lemma 7.1, we have

$$
\chi^{\underline{\lambda}} \downarrow G_{r} \times G_{n-r}=\sum_{|\underline{\mu}|=r} \chi^{\underline{\mu}} \times \chi^{\lambda / \mu} \underline{\mu} .
$$

If we apply Lemma 7.1 to $\chi^{\underline{\mu}} \downarrow G_{s} \times G_{r-s}$, we obtain

$$
\chi^{\underline{\lambda}} \downarrow G_{s} \times G_{r-s} \times G_{n-r}=\sum_{|\underline{\nu}|=s} \sum_{|\underline{\mu}|=r} \chi^{\underline{\nu}} \times \chi^{\underline{\mu}} \underline{\underline{\nu}} \times \chi^{\underline{\lambda}}-\underline{\mu} .
$$

However, if we apply Lemma 7.1 to $\chi^{\lambda} \downarrow G_{s} \times G_{n-s}$, we find

$$
\chi^{\underline{\lambda}} \downarrow G_{s} \times G_{r-s} \times G_{n-r}=\sum_{|\underline{\nu}|=s} \chi^{\nu}-\left(\chi^{\lambda / \nu}-\downarrow G_{r-s} \times G_{n-r}\right) .
$$

Compare the coefficients of $\chi^{\underline{\nu}}$.

If $\theta=\operatorname{char} U$ is a $G$-character and $\chi=\operatorname{char} V$ is an $S_{n}$-character, let $\theta<\chi:=\operatorname{char}(U \succ V)$ denote the corresponding $G_{n}$-character. To describe $\theta<\chi$ directly in terms of $\theta$ and $\chi$ is an easy exercise in linear algebra; one finds

LEMMA 7.3. If the cycles of $x \in G_{n}$ are of $G$-class $g_{1}, \ldots, g_{l}$ and $w$ is the $S_{n}$-image of $x$, then

$$
\theta \prec \chi(x)=\chi(w) \theta\left(g_{1}\right) \cdots \theta\left(g_{l}\right) .
$$

Proofs may be found in [JK, K].

LEMMA 7.4. Let $x \in G_{n}$ be an $n$-cycle of $G$-class $g$. We have $\chi^{\lambda}-\underline{\mu}(x)=0$ unless $\lambda / \mu$ is a skew hook of size $n$. In that case, if the character of $\underline{\lambda} \underline{\mu}$ is $\bar{\theta}$, then

$$
\chi^{\lambda /}-\underline{\mu}(x)=(-1)^{r-1} \theta(g),
$$

where $r$ is the number of nonempty rows in the diagram of $\underline{\lambda} \underline{\mu}$.

Proof. Recall that $\chi^{\lambda /} \underline{\underline{\mu}}$ is obtained via induction from a "Young" subgroup $G_{(n)}$. Using standard techniques for computing induced characters (e.g., $[\mathbf{C R},(10.3)]$, it follows that $\chi^{\lambda / \mu} \underline{\mu}(x)=0$ unless some $G_{n}$-conjugate of $x$ meets $G_{(n)}$. However, no such (proper) subgroup includes an $n$-cycle, so we only need to consider the case $G_{(n)}=G_{n}$. In that case, $\underline{\lambda}$ and $\underline{\mu}$ must be identical in every component but one, 
say the $\theta$ th, and we have $\chi^{\lambda /} \underline{\mu}=\theta \prec \chi$, where $\chi$ is the $S_{n}$-character indexed by $\lambda^{\theta} / \mu^{\theta}$.

Let $w \in S_{n}$ be an $n$-cycle. According to the Murnaghan-Nakayama rule $[\mathbf{J K}, 2.4]$, we have $\chi(w)=0$ unless $\lambda^{\theta} / \mu^{\theta}$ is a skew hook, and in that case, $\chi(w)=(-1)^{r-1}$, where $r$ is the number of rows in the diagram of $\lambda^{\theta} / \mu^{\theta}$. Apply Lemma 7.3.

Finally, to prove Theorem 4.3 , let $x=x_{1} \cdots x_{l}$ be a factorization of $x \in G_{n}$ into cycles of length $\beta_{1}, \ldots, \beta_{l}$ and $G$-class $g_{1}, \ldots, g_{l}$. If we regard $x$ as a member of the subgroup $G_{(\beta)}$, repeated application of Corollary 7.2 yields

$$
\chi^{\underline{\lambda}-\underline{\nu}}-(x)=\sum_{\left(\underline{\lambda}^{l}\right)} \chi^{\underline{\lambda}^{l} / \underline{\lambda}^{0}}\left(x_{1}\right) \cdots \chi^{\underline{\lambda}^{l} / \underline{\lambda}^{l-1}}\left(x_{l}\right),
$$

summed over all $\beta$-partitions $\left(\underline{\lambda}^{i}: 0 \leq i \leq l\right)$ of $\lambda / \nu$. The values of skew characters on the cycles $x_{i}$ may be evaluated via Lemma 7.4, thus yielding the description in Theorem 4.3.

B. The difference characters of $D_{n}$. Continuing the notation of $\S 6 \mathrm{C}$, let $\lambda$ be a partition of $n$, and let $\Delta^{\lambda}$ denote the difference character corresponding to the pair of irreducible $D_{2 n}$-constituents of the $B_{2 n}$ module indexed by $(\lambda, \lambda)$. The objective of this section is to derive the following explicit description of the $\Delta^{\lambda}$ 's, thus providing a rectification of Young's remarks in QSA V [Y].

THEOREM 7.5. Let $x \in D_{2 n}$.

(a) $\Delta^{\lambda}(x)=0$ unless every cycle of $x$ is of even length and of $C_{2}$-class +1 .

(b) If $x$ is a permutation matrix of cycle-type $2 \mu$, then

$$
\Delta^{\lambda}(x)=2^{\ell(\mu)} \chi^{\lambda}(\mu)
$$

To describe a difference character $\Delta$, it suffices to specify $\Delta(x)$ for one element $x \in D_{2 n}$ from each $B_{2 n}$-conjugacy class (Proposition 6.2). Thus, the description of $\Delta^{\lambda}$ given above is indeed complete.

Our proof follows the program outlined in $\S 6 \mathrm{~A}$. We first find a basis for the $B_{2 n}$-module indexed by $(\lambda, \lambda)$, and construct an associator $S$ (cf. (6.1)). The difference character may then be obtained from a straightforward trace calculation (cf. (6.4)). Using similar techniques it is possible to show that the DFT's of the irreducible characters of the reflection groups $G_{n}(d)\left(G=C_{m}\right)$ may be expressed in terms of the character tables of the reflection groups $C_{m / r}>S_{n / r}$ for $r \mid(m, n)$. 
$A$ basis for $(\lambda, \lambda)$. Let $V$ be an $S_{n}$-module. Eventually we will take $V$ to be the irreducible module indexed by $\lambda$, but this distinction will not be significant until later. Regard $V$ as a $B_{n}$-module via the obvious homomorphism $B_{n} \rightarrow S_{n}$, and use $V_{\delta}$ as an abbreviation for the $B_{n}$ module $\delta \otimes V$. (Recall that $\delta$ is the product-of-signs character from $\S 6 \mathrm{C})$.

Regard $B_{n} \times B_{n}$ as the subgroup of $B_{2 n}$ consisting of signed permutations of $\{1, \ldots, n\}$ and $\{n+1, \ldots, 2 n\}$.

A permutation $t \in S_{2 n}$ whose one-line notation $t_{1} \cdots t_{2 n}$ obeys $t_{1}<$ $\cdots<t_{n}$ and $t_{n+1}<\cdots<t_{2 n}$ will be called a tabloid, and we may depict these permutations as $2 \times n$ arrays:

$$
\left[\begin{array}{llll}
t_{1} & t_{2} & \ldots & t_{n} \\
t_{n+1} & t_{n+2} & \ldots & t_{2 n}
\end{array}\right]
$$

Let $T$ denote the abstract $\left(\begin{array}{c}2 n \\ n\end{array}\right)$-dimensional vector space spanned by tabloids. Since the tabloids form a set of coset representatives for $B_{2 n} / B_{n} \times B_{n}$, we may identify the vector space structure of $\left(V \otimes V_{\delta}\right) \uparrow$ $B_{2 n}$ with $T \otimes V \otimes V$. To transport the $B_{2 n}$-module structure to $T \otimes$ $V \otimes V$, choose a tabloid $t$, let $x \in B_{2 n}$, and suppose $x t=t^{\prime}\left(x_{1}, x_{2}\right)$, where $\left(x_{1}, x_{2}\right) \in B_{n} \times B_{n}$ and $t^{\prime}$ is the unique tabloid in the coset $x t\left(B_{n} \times B_{n}\right)$. Via the standard construction of induced representations, we may describe the action of $x$ on $T \otimes V \otimes V$ as follows:

$$
x\left(t \otimes v_{1} \otimes v_{2}\right)=\delta\left(x_{2}\right) t^{\prime} \otimes x_{1} v_{1} \otimes x_{2} v_{2} .
$$

In case $V$ is the irreducible $S_{n}$-module indexed by $\lambda$, this construction yields the irreducible $B_{2 n}$-module indexed by $(\lambda, \lambda)$ as defined in Theorem 4.1.

The associator. Let $t \mapsto \hat{t}$ denote the involution on tabloids obtained by swapping rows in (7.1), and define $S \in \mathrm{GL}(T \otimes V \otimes V)$ as follows:

$$
S\left(t \otimes v_{1} \otimes v_{2}\right)=\hat{t} \otimes v_{2} \otimes v_{1} .
$$

Note that $\hat{t}=t s$, where $s$ is the involution $(1, n+1)(2, n+2) \cdots(n, 2 n)$.

LEMMA 7.6. $S$ is an associator for $\left(V \otimes V_{\delta}\right) \uparrow B_{2 n}$.

Proof. Clearly $S$ is an involution. To prove that the defining condition (6.1) is satisfied, first consider the action of the diagonal matrix $y=\operatorname{diag}(1, \ldots, 1,-1) \in B_{2 n}$ on $T \otimes V \otimes V$. If $2 n$ occurs in row 1 of $t$, then (7.2) and (7.3) imply

$$
S y\left(t \otimes v_{1} \otimes v_{2}\right)=\hat{t} \otimes v_{2} \otimes v_{1},
$$


and if $2 n$ occurs in row 2 of $t$, then

$$
S y\left(t \otimes v_{1} \otimes v_{2}\right)=-\hat{t} \otimes v_{2} \otimes v_{1} .
$$

Since the row of $2 n$ changes under the operation $t \mapsto \hat{t}$, it follows that $(S y)^{2}=-1$ (or equivalently, $S y=-y S$ ) on $T \otimes V \otimes V$, as desired.

Now consider the action of permutations $x \in S_{2 n} \subset B_{2 n}$ on $T \otimes V \otimes$ $V$. In that case, note that $x t=t^{\prime}\left(x_{1}, x_{2}\right)$ for some tabloid $t^{\prime}$, where $x_{1,2} \in S_{n}$, and we have

$$
S x\left(t \otimes v_{1} \otimes v_{2}\right)=\left(t^{\prime}\right)^{\wedge} \otimes x_{2} v_{2} \otimes x_{1} v_{1} .
$$

Since $x \hat{t}=x t s=t^{\prime}\left(x_{1}, x_{2}\right) s=t^{\prime} s\left(x_{2}, x_{1}\right)=\left(t^{\prime}\right)^{\wedge}\left(x_{2}, x_{1}\right)$, it follows that $x S\left(t \otimes v_{1} \otimes v_{2}\right)=x\left(\hat{t} \otimes v_{2} \otimes v_{1}\right)=\left(t^{\prime}\right)^{\wedge} \otimes x_{2} v_{2} \otimes x_{1} v_{1}$,

and therefore $S x=x S$ on $T \otimes V \otimes V$. Since $B_{2 n}$ is generated by the permutations $x$ and the diagonal matrix $y$, the lemma follows.

We are now ready to give the

Proof of Theorem 7.5. Part (a) follows from Theorem 6.7(a), so we need only to consider the case in which $x \in D_{2 n}$ is a permutation matrix of cycle-type $2 \mu$. For convenience, we assume that the cycles of $x$ permute consecutive positions. For example, if $\mu=(3,1)$, we take $x=(1,2, \ldots, 6)(7,8)$.

As discussed above, if we take $V$ to be the irreducible $S_{n}$-module with character $\chi^{\lambda}$, then (6.4) implies

$$
\Delta^{\lambda}(x)=\operatorname{tr}\left(\left.S x\right|_{T \otimes V \otimes V}\right) .
$$

To evaluate this trace, choose a tabloid $t$ and a basis $v_{1}, v_{2}, \ldots$ for $V$. Let $\rho_{i j}(w)$ denote the matrix entries of the action of $w \in S_{n}$ on $V$ with respect to $v_{1}, v_{2}, \ldots$. Since $x$ is a pure permutation, we have $x t=t^{\prime}\left(x_{1}, x_{2}\right)$ for some tabloid $t^{\prime}$ and some $x_{1,2} \in S_{n}$. Moreover, (7.2) and (7.3) imply

$$
S x\left(t \otimes v_{i} \otimes v_{j}\right)=\left(t^{\prime}\right)^{\wedge} \otimes x_{2} v_{j} \otimes x_{1} v_{i}
$$

Therefore, in order for the basis vector $t \otimes v_{i} \otimes v_{j}$ to contribute to the trace, we must necessarily have $t=\left(t^{\prime}\right)^{\wedge}$; i.e., $t^{\prime}=\hat{t}$. Assuming $t$ to be such a tabloid, observe that the subspace $\mathbf{C} t \otimes V \otimes V$ contributes

$$
\sum_{i, j} \rho_{j i}\left(x_{2}\right) \rho_{i j}\left(x_{1}\right)=\operatorname{tr} \rho\left(x_{1} x_{2}\right)=\chi^{\lambda}\left(x_{1} x_{2}\right)
$$

to the trace. 
To understand the condition $t^{\prime}=\hat{t}$, suppose, for example, that $x_{0}=(1,2, \ldots, 6)$ is a cycle of $x$. In that case, it is not hard to see that $t^{\prime}=\hat{t}$ forces $\{1,3,5\}$ and $\{2,4,6\}$ to appear in opposite rows of $t$. In particular, there are only two possible choices for the subtabloid $t_{0}$ occupied by $\{1, \ldots, 6\}$ : either $t_{0}=\left[\begin{array}{lll}1 & 3 & 5 \\ 2 & 4 & 6\end{array}\right]$, or $t_{0}=\left[\begin{array}{lll}2 & 4 & 6 \\ 1 & 3 & 5\end{array}\right]$. Furthermore, in these respective cases, we find either $x_{0} t_{0}=\hat{t}_{0}(1,(321))$ or $x_{0} t_{0}=\hat{t}_{0}((321), 1)$.

In general, there are a total of $2^{\ell(\mu)}$ tabloids $t$ such that $t^{\prime}=\hat{t}$, and each cycle of $x$, say $(2 a+1, \ldots, 2 b)$, contributes a cycle $(b, b-1, \ldots, a+$ 1) to either $x_{1}$ or $x_{2}$. Regardless of how the cycles are distributed to $x_{1}$ or $x_{2}$, it follows that $x_{1} x_{2} \in S_{n}$ is of cycle-type $\mu$. Hence, (7.4) shows that each of the $2^{\ell(\mu)}$ choices for $t$ contributes $\chi^{\lambda}(\mu)$ to $\Delta^{\lambda}(x)$. $\square$

We remark that the vectors $t \otimes v_{i} \otimes v_{j} \pm \hat{t} \otimes v_{j} \otimes v_{i}$ are clearly eigenvectors for $S$, and thus provide explicit bases for the irreducible $D_{2 n}$-modules indexed by $\{\lambda, \lambda\}_{ \pm}$.

\section{REFERENCES}

[B] N. Bourbaki, Groupes et Algèbres de Lie, Chp. IV,V,VI, Hermann, Paris, 1971.

[C] R. W. Carter, Finite Groups of Lie Type: Conjugacy Classes and Complex Characters, Wiley, New York, 1985.

[Ch] C. Chevalley, Invariants of finite groups generated by reflections, Amer. J. Math., 77 (1955), 778-782.

[Col] A. J. Coleman, The Betti numbers of the simple Lie groups, Canad. J. Math., 10 (1958), 349-356.

[Co] H. S. M. Coxeter, The product of the generators of a finite group generated by reflections, Duke Math. J., 18 (1951), 765-782.

[CR] C. W. Curtis and I. Reiner, Methods of Representation Theory, Vol. I, Wiley, New York, 1981.

[G] A. M. Garsia, Combinatorics of the free Lie algebra and the symmetric group, preprint.

[GW] A. M. Garsia and M. Wachs, Combinatorial aspects of skew representations of the symmetric group, J. Combin. Theory Ser. A, to appear.

[JK] G. D. James and A. Kerber, The Representation Theory of the Symmetric Group, Addison-Wesley, Reading, MA, 1981.

[J] A. Joyal, Foncteurs analytiques et espèces de structures, in Combinatoire Énumérative, (G. Labelle and P. Leroux, eds.), Lecture Notes in Math., Vol. 1234, Springer, Berlin, 1986, pp.126-159.

[K] A. Kerber, Representations of Permutation Groups, I, II, Springer Lecture Notes in Math., Vols. 240, 495, Springer, Berlin, 1971, 1975.

[KW] W. Kraśkiewicz and J. Weyman, Algebras of coinvariants and the action of Coxeter elements, preprint.

[L] G. Lusztig, Irreducible representations of finite classical groups, Invent. Math., 43 (1977), 125-175. 
[M1] I. G. Macdonald, Symmetric Functions and Hall Polynomials, Oxford Univ. Press, Oxford, 1979.

[M2] I. G. Macdonald, Polynomial functors and wreath products, J. Pure Appl. Algebra, 18 (1980), 173-204.

[ST] G. C. Shephard and J. A. Todd, Finite unitary reflection groups, Canad. J. Math., 6 (1954), 274-304.

[Spe] W. Specht, Eine Verallgemeinerung der symmetrischen Gruppe, Schriften Math. Seminar (Berlin), 1 (1932), 1-32.

[Sp] T. A. Springer, Regular elements of finite reflection groups, Invent. Math., 25 (1974), 159-198.

[St1] R. P. Stanley, Invariants of finite groups and their applications to combinatorics, Bull. Amer. Math. Soc., 1 (1979), 475-511.

[St2] R. P. Stanley, Some aspects of groups acting on finite posets, J. Combin. Theory Ser. A, 32 (1982), 132-161.

[St3] R. P. Stanley, private communication.

[S1] R. Steinberg, A geometric approach to the representations of the full linear group over a Galois field, Trans. Amer. Math. Soc., 71 (1951), 274-282.

[S2] R. Steinberg, Finite reflection groups, Trans. Amer. Math. Soc., 91 (1959), 493-504.

[Y] A. Young, On quantitative substitutional analysis (QSA V), Proc. London Math. Soc., 31 (1930), 273-288.

Received April 6, 1988. Partially supported by NSF grant number DMS-8603228. The author was also supported during part of the research by the Institute for Mathematics and its Applications with funds provided by the NSF.

UNIVERSITY OF MICHIGAN

ANN ARBOR, MI 48109 


\section{PACIFIC JOURNAL OF MATHEMATICS EDITORS}

\author{
V. S. VARAdarajan \\ (Managing Editor) \\ University of California \\ Los Angeles, CA 90024-1555-05 \\ Herbert Clemens \\ University of Utah \\ Salt Lake City, UT 84112 \\ ThOMAS ENRIGHT \\ University of California, San Diego \\ La Jolla, CA 92093
}

R. FINN

Stanford University

Stanford, CA 94305

HeRmann FlaschKa

University of Arizona

Tucson, AZ 85721

VAUGHAN F. R. Jones

University of California

Berkeley, CA 94720

SteVen KercKhofF

Stanford University

Stanford, CA 94305

\section{ROBION KIRBY}

University of California

Berkeley, CA 94720

C. C. MOORE

University of California

Berkeley, CA 94720

HAROLD STARK

University of California, San Diego

La Jolla, CA 92093

\section{ASSOCIATE EDITORS}
R. Arens
E. F. BeCKenbaCH
B. H. NEUMANN
F. Wolf
K. YoshidA (1906-1982)

\section{SUPPORTING INSTITUTIONS}

UNIVERSITY OF ARIZONA

UNIVERSITY OF BRITISH COLUMBIA

CALIFORNIA INSTITUTE OF TECHNOLOGY

UNIVERSITY OF CALIFORNIA

MONTANA STATE UNIVERSITY

UNIVERSITY OF NEVADA, RENO

NEW MEXICO STATE UNIVERSITY OREGON STATE UNIVERSITY

\author{
UNIVERSITY OF OREGON \\ UNIVERSITY OF SOUTHERN CALIFORNIA \\ STANFORD UNIVERSITY \\ UNIVERSITY OF HAWAII \\ UNIVERSITY OF TOKYO \\ UNIVERSITY OF UTAH \\ WASHINGTON STATE UNIVERSITY \\ UNIVERSITY OF WASHINGTON
}

The Supporting Institutions listed above contribute to the cost of publication of this Journal, but they are not owners or publishers and have no responsibility for its content or policies.

Mathematical papers intended for publication in the Pacific Journal of Mathematics should be in typed form or offset-reproduced (not dittoed), double spaced with large margins. Please do not use built up fractions in the text of the manuscript. However, you may use them in the displayed equations. Underline Greek letters in red, German in green, and script in blue. The first paragraph must be capable of being used separately as a synopsis of the entire paper. In particular it should contain no bibliographic references. Please propose a heading for the odd numbered pages of less than 35 characters. Manuscripts, in triplicate, may be sent to any one of the editors. Please classify according to the scheme of Math. Reviews, Index to Vol. 39. Supply name and address of author to whom proofs should be sent. All other communications should be addressed to the managing editor, or Elaine Barth, University of California, Los Angeles, California 90024-1555-05.

There are page-charges associated with articles appearing in the Pacific Journal of Mathematics. These charges are expected to be paid by the author's University, Government Agency or Company. If the author or authors do not have access to such Institutional support these charges are waived. Single authors will receive 50 free reprints; joint authors will receive a total of 100 free reprints. Additional copies may be obtained at cost in multiples of 50 .

The Pacific Journal of Mathematics is issued monthly as of January 1966. Regular subscription rate: $\$ 190.00$ a year (5 Vols., 10 issues). Special rate: $\$ 95.00$ a year to individual members of supporting institutions.

Subscriptions, orders for numbers issued in the last three calendar years, and changes of address should be sent to Pacific Journal of Mathematics, P.O. Box 969, Carmel Valley, CA 93924, U.S.A. Old back numbers obtainable from Kraus Periodicals Co., Route 100, Millwood, NY 10546.

The Pacific Journal of Mathematics at P.O. Box 969, Carmel Valley, CA 93924 (ISSN 0030-8730) publishes 5 volumes per year. Application to mail at Second-class postage rates is pending at Carmel Valley, California, and additional mailing offices. Postmaster: send address changes to Pacific Journal of Mathematics, P.O. Box 969, Carmel Valley, CA 93924.

PUBLISHED BY PACIFIC JOURNAL OF MATHEMATICS, A NON-PROFIT CORPORATION Copyright (C) 1989 by Pacific Journal of Mathematics 


\section{Pacific Journal of Mathematics \\ Vol. 140, No. $2 \quad$ October, 1989}

Edoardo Ballico, Spanned and ample vector bundles with low Chern numbers

Marcy Mason Barge, Richard Swanson and Russell Bruce Walker,

Conjugacy class structure of smooth hyperbolic sectors . ........... 217

Jeffrey Stephen Fox, Adeles and the spectrum of compact nilmanifolds . . ..233

Robert D. Little, Homotopy complex projective spaces with divisible

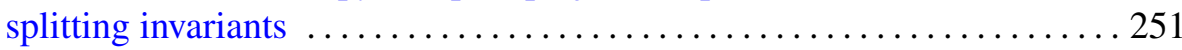

M. Scott Osborne and Garth William Warner, Jr., The Selberg trace formula. VII. Application of the truncation process to the continuous

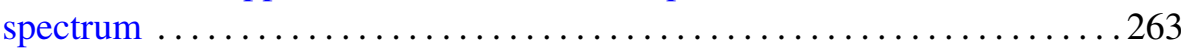

John R. Stembridge, On the eigenvalues of representations of reflection

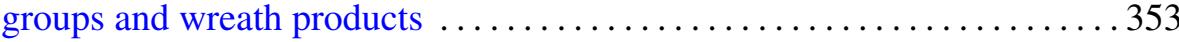

Ibrahim Salama, Corrections to: "Topological entropy and recurrence of

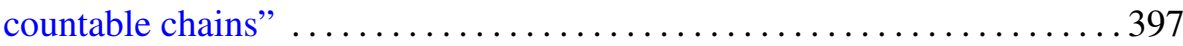

Robert Greene and Hung-Hsi Wu, Addendum to: "Lipschitz convergence of Riemannian manifolds" ............................... 398

Kayoko Shikishima-Tsuji, Correction to: "Galois theory of differential fields of positive characteristic" 